\title{
Minor axis kinematics of 19 S0-Sbc bulges ${ }^{\star}$
}

\author{
J. Falcón-Barroso ${ }^{1}$, M. Balcells ${ }^{2}$, R. F. Peletier ${ }^{1,3}$, and A. Vazdekis ${ }^{2}$ \\ 1 School of Physics \& Astronomy, University of Nottingham, Nottingham NG7 2RD, UK \\ e-mail: Reynier.Peletier@nottingham.ac.uk \\ 2 Instituto de Astrofísica de Canarias, 38200 La Laguna, Tenerife, Spain \\ e-mail: balcells@1l.iac.es; vazdekis@ll.iac.es \\ 3 CRAL, Observatoire de Lyon, 69561 St-Genis Laval cedex, France
}

Received 15 November 2002 / Accepted 18 March 2003

\begin{abstract}
We present minor axis kinematic profiles for a well-studied sample of 19 early- to intermediate-type disk galaxies. We introduce, for the first time, the use of single-burst stellar population (SSP) models to obtain stellar velocities, velocity dispersions and higher order Gauss-Hermite moments $\left(h_{3}, h_{4}\right)$ from galaxy spectra in the near-infrared Ca II triplet region. SSP models, which employs the synthetic spectra of Vazdekis et al. (2003), provide a means to address the template-mismatch problem, and are shown to provide as good or better fits as traditional stellar templates. We anticipate the technique to be of particular use for high-redshift galaxy kinematics. We give the measurement of a recently defined $\mathrm{CaT}^{*}$ index (Cenarro et al. 2001a), and describe the global properties of the bulge kinematics as derived from the kinematic profiles. We detect smallamplitude minor-axis rotation, generally due to inner isophotal twists as a result of slightly triaxial bulges or misaligned inner disks; such inner features do not show peculiar colors or distinct $\mathrm{CaT}^{*}$ index values. Velocity dispersion profiles, which extend well into the disk region, show a wide range of slopes. Flattened bulges tend to have shallower velocity dispersion profiles. The inferred similarity of bulge and disk radial velocity dispersions supports the interpretation of these bulges as thickened disks.
\end{abstract}

Key words. galaxies: bulges - galaxies: kinematics and dynamics - galaxies: structure - methods: data analysis

\section{Introduction}

The measurement of kinematics in galaxies has been one of the major achievements in astrophysics in the second half of the last century. Our knowledge in this field has evolved from crude kinematic determinations from photographic plates (Burbidge $\&$ Burbidge 1968), to high-resolution, detailed measurements of the line-of-sight velocity distributions (hereafter LOSVDs; i.e. Bender et al. 1994; Fisher 1997; Vega Beltrán et al. 2001).

In the seventies, methods were devised to measure velocity dispersions in addition to radial velocities, i.e. cross-correlation (Tonry \& Davis 1979), Fourier Quotient (Sargent et al. 1977). In the past decade, with the improved quality of the data, a new set of algorithms were developed allowing us to measure LOSVDs (Fourier Correlation Quotient, Bender 1990), and quantify deviations from pure single Gaussian (van der Marel \& Franx 1993) and multiple Gaussian LOSVD with UGD (Kuijken \& Merrifield 1993; Prada et al. 1996). Galactic kinematics, however, suffers from an important technical constraint: template mismatching. Stars are observed together with

\footnotetext{
Send offprint requests to: J. Falcón-Barroso,

e-mail: ppxjf2@nottingham.ac.uk

* Appendix B is only available in electronic form at http://www.edpsciences.org
}

the object which kinematics we are interested in, to be used as spectral templates. However, it is difficult to choose the right star, since galaxy light comes from a wide range of stellar types. Although, for ellipticals in the $V$-band, it has been found that half of the light comes from $G$ and $K$ giants (Pickles 1985) and, therefore, those stellar types are good templates, this situation may change dramatically from galaxy to galaxy or even within a galaxy. Therefore kinematic parameters will be sensitive to the stellar template used. Much work has been devoted to address the template mismatch problem (Rix \& White 1992; González 1993; Statler et al. 1996; Statler \& Smecker-Hane 1999).

In the last decade, new evolutionary stellar population synthesis models (Bruzual A. \& Charlot 1993; Worthey et al. 1994; Buzzoni 1995; Tantalo et al. 1996; Vazdekis et al. 1996) have helped us to significantly increase our galaxy stellar populations knowledge. These models predict line strengths of a limited number of spectral features, such as those of the Lick/IDS system (i.e. Worthey et al. 1994; Vazdekis et al. 1996). However since these models were unable to predict full SEDs (Spectral Energy Distributions) at high enough resolution, they could not be used as templates for galaxy stellar kinematics. This situation has recently changed with the development of new SSP model SEDs in the blue and visual at 
resolution $1.8 \AA$ ( $F W H M$ ) by Vazdekis (1999) and in the nearIR Ca II triplet region at $1.5 \AA$ (Vazdekis et al. 2003). In fact, Vazdekis (1999) and Vazdekis \& Arimoto (1999) have shown the potential use of the SSP models as templates for determining the main kinematic parameters in the optical spectral range. In this paper we are for the first time in position to determine galaxy kinematics using SSP templates in the near-IR Ca II spectral range. It is generally assumed, that kinematic stellar templates are required to be obtained with the same instrumental setting as the one used for the galaxy spectra (Sargent et al. 1977; Tonry \& Davis 1979). In this paper we show that this is no longer a requirement, at least at the resolutions of the spectra considered here.

This paper intends to be a first step in the exploration of SSP models as templates by comparing velocity profiles, for a sample of 19 galaxies, with those obtained with our best stellar or mixture of stellar templates. We show that in all cases SSP models produce kinematics of at least at the same level of quality, and are quite often better than the best stellar templates. In order to trace correlations between kinematic and population features along the galaxy minor axes, we measure the $\mathrm{CaT}^{*}$ index (Cenarro et al. 2001a).

Section 2 summarizes the observations and data reduction. In Sect. 3 we describe the different types of template samples and the way the kinematical profiles have been determined, including a detailed comparison between stellar templates and SSP models. The obtained kinematics is discussed in Sect. 4. Kinematical profiles are graphically presented in Appendix A. Tables containing the numerical values presented in Appendix A will be available in electronic form.

\section{Observations and data reduction}

\subsection{Sample}

The sample comprises 20 galaxies of types $\mathrm{S} 0-\mathrm{Sbc}$ selected out of the Balcells \& Peletier (1994) sample of bulges. The latter is a diameter-limited sample of galaxies with inclinations above 50 degree from the UGC catalog (Nilson 1995); it is has been the subject of several studies on bulges (see Falcón-Barroso et al. 2002 and references therein). The 20 objects were those we had observed with HST/WFPC2 and NICMOS, hence highspatial resolution images are available for them at $B, I, H$ bands (Peletier et al. 1999). The main properties of the sample are presented in Table 1.

\subsection{Observations}

We obtained long-slit spectra for our sample of galaxies at the 4.2-m William Herschel Telescope of the Observatorio del Roque de los Muchachos at La Palma between 11 and 13 July 1997. Twenty galaxies were observed between 8360-9170 $\AA$ using the ISIS double spectrograph. We discarded NGC 5577 as the spectra did not have sufficient signal-to-noise. We are thus left with 19 objects (see Table 1). The red arm was equipped with the Tektronix $(1024 \times 1024)$ TEK2 CCD $\left(0.36^{\prime \prime}\right.$ per $24 \mu \mathrm{m}$ pixel) and the R600R grating, providing a spectral resolution (FWHM of arc lines) of $1.74 \AA\left(59.6 \mathrm{~km} \mathrm{~s}^{-1}\right)$ with a spectral dispersion of $0.8 \AA /$ pix. We used the $6100 \AA$ dichroic for simultaneous red and blue arm exposures. In this paper we discuss the red arm spectra only. Typical exposure times per galaxy were $1500 \mathrm{~s}$. The length of the slit was $3.6^{\prime}$. The slit width was $1.2^{\prime \prime}$, matching the seeing at the time of the observations. Arc line exposures were taken before and after each target exposure. Tungsten continuum lamp exposures were taken with the red arm after each target exposure, for fringe calibration. Twilight sky exposures were taken every night for flat fielding. We also obtained spectra for 11 stellar templates of types A2 to M4, from the Lick list of stars (Worthey et al. 1994), listed in Table 2.

\subsection{Data reduction}

The data reduction was carried out using the $\mathrm{IRAF}^{1}$ package. The general standard procedures were applied to the data (bias subtraction, flat fielding, fringe correction, sky subtraction, wavelength and flux calibration), as described in Falcón-Barroso et al. (2002). Cosmic ray removal was performed using the $\mathrm{RED}_{\mathrm{m}}^{\mathrm{uc}} \mathrm{E}$ package (Cardiel 1999).

\section{Data analysis}

\subsection{Stellar templates}

In Table 2 we list the observed stellar templates. A representative set of spectra is shown in Fig. 1. The wide range of spectral types covered allows us for an exploration of the best templates for matching galaxy spectra. The most apparent variation from top to bottom in Fig. 1 is the decrease of the Paschen lines toward later types. P14, in a region devoid of other features, is a strong discriminant of the presence of stars earlier than G. P13 and P15, when present, give characteristic asymmetric profiles to the second and third lines of the CaII (Cenarro et al. 2001b). Following previous works in the literature (Rix \& White 1992; Hau et al. 1999) we have included in our analysis a mix-template from a linear combination of stars in our sample. However we have used a different approach to derive our optimal mix-template. Given the importance of the continuum shape in this wavelength region (Cenarro et al. 2001a; Vazdekis et al. 2003), we have created an optimal template that provides the best fit to the overall continuum shape of each galaxy (i.e. fitting the regions outside the main Ca II features, but including the $\mathrm{TiO}$ bands), before broadening with the appropriate kinematics. It is important to perform this fit using a combination of unconvolved stellar spectra, since any input kinematics comes from a particular template and could bias our result. In most cases the optimal mix-template is formed by G8III, K3Ib and M4V stars. The importance of the M4V star in the final template is justified by its ability to match the $\mathrm{TiO}$ molecular bands. As we will show in Sect. 3.4, this mixture of stellar templates helps to alleviate the template mismatch problem and it is an ideal benchmark to test our SSP models.

\footnotetext{
${ }^{1}$ IRAF is distributed by the National Optical Astronomy Observatories, which are operated by the Association of Universities for Research in Astronomy, Inc., under cooperative agreement with the National Science Foundation.
} 
Table 1. Galaxy sample.

\begin{tabular}{|c|c|c|c|c|c|c|c|c|c|c|c|c|c|}
\hline $\begin{array}{l}\text { NGC } \\
(1)\end{array}$ & $\begin{array}{c}V_{\mathrm{LG}} \\
\mathrm{km} \mathrm{s}^{-1} \\
(2)\end{array}$ & $\begin{array}{c}\text { Scale } \\
\mathrm{kpc} /{ }^{\prime \prime} \\
(3)\end{array}$ & $\begin{array}{r}\text { Type } \\
\text { (4) }\end{array}$ & $\begin{array}{c}M_{R}^{\mathrm{tot}} \\
\operatorname{mag} \\
(5)\end{array}$ & $\begin{array}{c}M_{R}^{\mathrm{bul}} \\
\mathrm{mag} \\
(6)\end{array}$ & $\begin{array}{l}B / D \\
(7)\end{array}$ & $\begin{array}{r}\text { PA } \\
\text { deg } \\
(8)\end{array}$ & $\begin{array}{c}\text { Exp. time } \\
\text { s } \\
(9)\end{array}$ & $\begin{array}{l}\epsilon_{\mathrm{D}} \\
(10)\end{array}$ & $\begin{array}{l}\epsilon_{\mathrm{B}} \\
(11)\end{array}$ & $\begin{array}{c}\mathrm{r}_{\text {bulge }} \\
\text { " } \\
(12)\end{array}$ & Best templ. & $\begin{array}{c}S / N \\
\operatorname{pix}^{-1} \\
(14)\end{array}$ \\
\hline 5326 & 2576 & 0.25 & 1 & -22.16 & -21.22 & 0.73 & -138 & 1500 & 0.50 & 0.43 & 5.97 & $+0.2,3.16$ & 83 \\
\hline 5389 & 1990 & 0.19 & 0 & -21.32 & -20.70 & 1.30 & 93 & 1500 & 0.80 & 0.46 & 2.85 & M1 III / +0.2, 4.47 & 61 \\
\hline 5422 & 1929 & 0.19 & -2 & -21.96 & -21.33 & 1.28 & 64 & 1500 & 0.80 & 0.35 & 3.33 & $+0.2,7.08$ & 68 \\
\hline 5443 & 2060 & 0.20 & 3 & -21.53 & -19.09 & 0.12 & -52 & 1400 & 0.68 & 0.54 & 1.98 & $+0.2,3.16$ & 37 \\
\hline 5475 & 1861 & 0.18 & 0 & -20.98 & -18.72 & 0.14 & -101 & 1500 & 0.68 & 0.24 & 2.45 & $+0.2,1.41$ & 57 \\
\hline 5587 & 2291 & 0.22 & 0 & -21.07 & -19.43 & 0.29 & 73 & 1500 & 0.70 & 0.47 & 1.51 & $-0.4,14.12$ & 36 \\
\hline 5689 & 2290 & 0.22 & 0 & -22.29 & -21.47 & 0.89 & -5 & 1500 & 0.75 & 0.48 & 2.04 & $0.0,4.47$ & 58 \\
\hline 5707 & 2354 & 0.23 & 2 & -21.43 & -20.11 & 0.42 & -59 & 1500 & 0.75 & 0.20 & 3.79 & $0.0,2.51$ & 51 \\
\hline 5719 & 1684 & 0.16 & 2 & -21.60 & -20.00 & 0.30 & 5 & 1500 & 0.64 & 0.61 & 7.71 & $\mathrm{~K} 3 \mathrm{Ib} /-0.4,3.55$ & 51 \\
\hline 5746 & 1677 & 0.16 & 3 & -22.68 & -21.70 & 0.68 & -99 & 1200 & 0.84 & 0.52 & 2.37 & M1 III / +0.2, 3.16 & 44 \\
\hline 5838 & 1337 & 0.13 & -3 & -21.89 & -20.93 & 0.71 & 134 & 1500 & 0.65 & 0.22 & 8.39 & $0.0,12.59$ & 86 \\
\hline 5854 & 1708 & 0.17 & -1 & -21.43 & -20.18 & 0.46 & -33 & 1500 & 0.70 & 0.32 & 4.85 & $-0.7,6.31$ & 64 \\
\hline 5879 & 1065 & 0.10 & 4 & -20.30 & -19.08 & 0.48 & 89 & 1500 & 0.62 & 0.32 & 0.85 & $-0.4,3.16$ & 41 \\
\hline 5965 & 3603 & 0.35 & 3 & -22.92 & -21.76 & 0.53 & -38 & 1500 & 0.84 & 0.50 & 2.32 & $-0.4,5.01$ & 46 \\
\hline 6010 & 1923 & 0.19 & 0 & -21.57 & -19.88 & 0.27 & 12 & 1500 & 0.75 & 0.28 & 2.70 & $0.0,14.12$ & 67 \\
\hline 6504 & 4680 & 0.46 & 2 & -24.57 & -23.30 & 0.45 & 135 & 1500 & 0.83 & 0.44 & 4.05 & $-0.4,3.16$ & 52 \\
\hline 7331 & 1138 & 0.11 & 3 & - & - & $0.001^{1}$ & 80 & 1500 & $0.63^{1}$ & 0.62 & 4.57 & $-0.4,15.85$ & 117 \\
\hline 7332 & 1550 & 0.15 & -2 & -21.86 & -20.52 & 0.41 & -114 & 1500 & 0.74 & 0.29 & 2.99 & $-0.4,11.22$ & 101 \\
\hline 7457 & 1114 & 0.11 & -3 & -20.91 & -20.91 & 10.00 & 35 & 1500 & 0.48 & 0.34 & 3.29 & $+0.2,2.51$ & 51 \\
\hline
\end{tabular}

Notes: Description of the columns:

(1): The NGC numbers of the galaxies.

(2): Recession velocity of each galaxy in $\mathrm{km} \mathrm{s}^{-1}$, corrected to the Local Group (Karachentsev \& Makarov 1996), from optical heliocentric velocities given in the Third Reference Catalogue of Bright Galaxies (1991) (hereafter RC3). (3): Scale in kpc/arcsec from Falcón-Barroso et al. (2002).

(4): Type index $T$ from the RC3.

(5) and (6): Absolute magnitudes, in $R$-band, from Peletier \& Balcells (1997) $\left(H_{0}=50 \mathrm{~km} \mathrm{~s}^{-1} \mathrm{Mpc}^{-1}\right)$.

(7): Bulge-to-disk ratio from an $R$-band bulge-disk decomposition following Kent's (1984) method (Peletier \& Balcells 1997).

(8): The position angle, in degrees, (N-E) of the dust-free minor axis (Peletier \& Balcells 1997).

(9): Integration time, in sec, for each galaxy during the observations.

(10): Disk ellipticity from Peletier \& Balcells (1997).

(11): Effective bulge ellipticity, from HST $H$-band ellipticity profiles, measured at the geometric effective radius (Balcells et al. 2003).

(12): Minor axis bulge radius, derived from Balcells et al. (2003) HST $H$-band profiles, as the radius at which the contribution from the disk and bulge light are equal, except for NGC 7332 for which a ground-based image was used instead (Peletier \& Balcells 1997) (Alister W. Graham, private communication).

(13): Best template fit for each galaxy. The metallicity $([\mathrm{m} / \mathrm{H}])$ and age $(\mathrm{Gyr})$ of the best fit SSP models is shown. In those cases where the stellar template performed better, the adopted SSP best template is also given.

(14): Signal-to-noise ratio per pixel in the central aperture $\left(1.2 \times 0.36 \operatorname{arcsec}^{2}\right)$.

${ }^{1}$ From Prada et al. (1996).

\subsection{SSP model templates}

We have used 78 SSP synthetic models at $F W H M=1.5 \AA$ $(0.85 \AA / p i x)$ in the Ca II triplet region, corresponding to a single-age, single-metallicity stellar populations of $[\mathrm{Fe} / \mathrm{H}]=$ $-0.7,-0.4,0.0$, and +0.2 . For each metallicity, the age varies from 1 to $17 \mathrm{Gyr}$, except for $[\mathrm{Fe} / \mathrm{H}]=-0.7$ starting from $6 \mathrm{Gyr}$ and for $[\mathrm{Fe} / \mathrm{H}]=-0.4$ starting $3 \mathrm{Gyr}$. These limitations arise from the limited coverage of atmospheric parameters of the stellar sample used by the models. More details can be found in Vazdekis et al. (2003). In order to adapt these models to the instrumental resolution of our data, we have convolved them with a Gaussian of $\sigma=0.38 \AA$. This procedure relies on the assumption that a Gaussian represents a good match to the instrumental PSF (Point Spread Function) of the observations. We have tested whether the arclines followed a Gaussian shape, finding that indeed they do, with only small deviations (below 0.01) in the $h_{3}$ and $h_{4}$ parameters, as described by van der Marel \& Franx (1993). If $h_{3}$ and $h_{4}$ had been found to be significantly different from 0 , we would have had to convolve the models with the line profile of the arc-lines.

\subsection{Extraction of the kinematics}

Kinematic profiles were derived from the data in the region of the near-IR Ca II triplet ( $8498 \AA$ А, $8542 \AA, 8662 \AA)$. For each galaxy, we generated two sets of coadded galaxy spectra, with 
Table 2. Stellar templates.

\begin{tabular}{|c|c|c|c|c|}
\hline $\begin{array}{l}\text { Name } \\
\text { (1) }\end{array}$ & $\begin{array}{c}\alpha(\mathrm{h} \mathrm{m} \mathrm{s}) \\
(2)\end{array}$ & $\begin{array}{c}\delta(\mathrm{d} \mathrm{m} \mathrm{s}) \\
(3)\end{array}$ & $\begin{array}{c}\text { Spectral type } \\
\text { (4) }\end{array}$ & $\begin{array}{r}M_{V} \\
(5)\end{array}$ \\
\hline BARNARD $^{1}$ & 175748.50 & +044136.2 & M4 Ve & 9.54 \\
\hline GL818 ${ }^{2}$ & 210519.75 & +070409.5 & K6 V & 8.33 \\
\hline HR0072 & 001841.87 & -080310.8 & G0 V & 6.46 \\
\hline HR4521 & 114655.62 & +553741.5 & K3 III & 5.26 \\
\hline HR5340 & 141539.67 & +191056.7 & $\mathrm{K} 3 \mathrm{Ib}$ & 1.23 \\
\hline HR5681 & 151530.16 & +331853.4 & G8 III & 3.47 \\
\hline HR5826 & 153124.93 & +772057.7 & K5 III & 5.00 \\
\hline HR5854 & 154416.07 & +062532.3 & K2 III & 2.64 \\
\hline HR6685 & 175525.19 & +260300.0 & F2 Ibe & 5.47 \\
\hline HR8334 & 214526.93 & +610714.9 & A2 Iab & 4.31 \\
\hline HR8795 & 230700.26 & +092434.2 & M1 III & 4.55 \\
\hline
\end{tabular}

Notes:

(1) HR names from the Bright Star Catalogue (Hoffleit \& Warren 1995).

(2),(3) J2000 coordinates from SIMBAD.

(4) spectral type from Worthey et al. (1994).

(5) from SIMBAD (http://simbad.u-strasbg.fr/Simbad).

1 NSV 9910 from the New catalogue of suspected variable stars (Kukarkin \& Kholopov 1982).

${ }^{2}$ HD 200779 from the Henry Draper Catalogue and Extension (Cannon \& Pickering 1993).

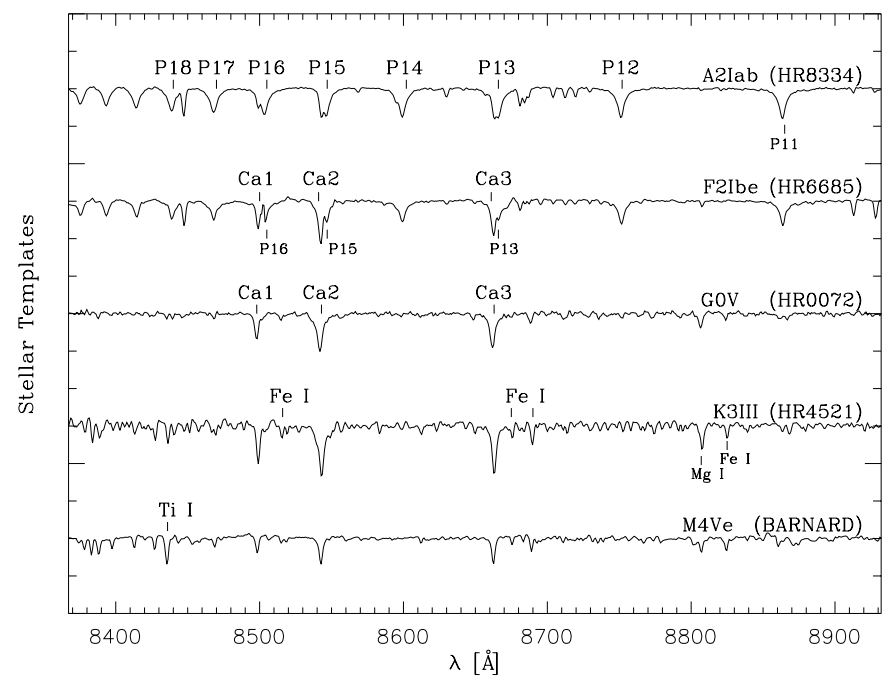

Fig. 1. Representative sample of continuum subtracted stellar templates used in this paper. The most important features found in those spectra have been indicated: the Paschen series (from P11 to P18), the $\mathrm{Ca}$ II triplet (Ca1, Ca2 and $\mathrm{Ca} 3$ ) and several metal lines (Fe I, Mg I and Ti I).

minimum signal-to-noise ratios $(S / N)$ of 33 and 20 per pixel, respectively. We made use of the program FOURFIT developed by van der Marel \& Franx (1993), available from the first author's website. A Gauss-Hermite fit to the LOSVD was performed to obtain mean radial velocities, velocity dispersions, $h_{3}$ and $h_{4}$ moments for the data with signal-to-noise ratio above

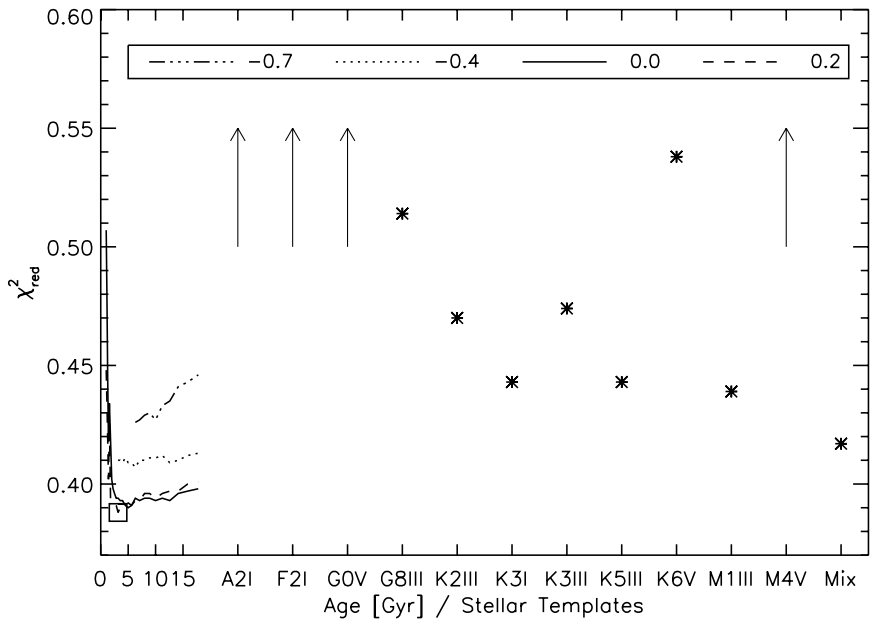

Fig. 2. $\chi^{2}$ distribution for NGC 5326. Open square indicates the minimum $\chi^{2}$ for the best SSP fitting model, from which kinematics has been derived for this galaxy. $\chi^{2}$ values for the stellar templates A2 Iab, F2 Ibe, G0 V and M4 Ve have been plotted with arrows indicating that they fall outside the plot limits. Their $\chi^{2}$ values are: $2.197,1.313$, $0.613,1.715$ respectively.

33 per pixel. For data with signal-to-noise ratio between 33 and 20 per pixel a pure Gaussian fit was used. The choice of $S / N$ is explained in Sect. 3.5.

\subsection{Selection of the best fitting template}

We have determined the kinematic profiles for our sample using each of the 90 templates we had available (78 SSP models, 11 stellar templates and the best mix-template for each galaxy). We have considered as our best fit template the one that minimized the $\chi^{2}$, as defined in van der Marel \& Franx (1993), for the central position of the galaxy profile, which has a $S / N$ well above 33. One of the features of this code is the possibility of filtering the input spectra in Fourier space so that any remaining low-order component not removed by the continuum subtraction or high-frequency noise can be filtered out. The resulting $\chi^{2}$ is then evaluated over a finite range of wavenumbers determined by the filtering parameters. For our dataset the results from FOURFIT were weakly dependent on the lower cutoff and insensitive to the choice of the upper cutoff (given that we chose the same upper cutoff for all the galaxies and that this cut was well above the sigma of the galaxy). We also reduce the uncertainties in the derived kinematics by fluxcalibrating the data and the template stars before applying the continuum subtraction because the normalizing polynomia for the data should be very similar to the ones used for the templates. otherwise, the normalization procedure might introduce non-negligible variations in the spectra, including in the equivalent widths of the lines.

We establish whether SSP, stellar templates or mixtemplate provide better fits to the galaxy spectra by comparing the global reduced $\chi^{2}$ of the fits. A typical distribution of $\chi^{2}$ values is shown in Fig. 2. The lines trace reduced $\chi^{2}$ for the SSP models, labeled by age and metallicity, while the crosses are the reduced $\chi^{2}$ of the stellar templates labeled by their 


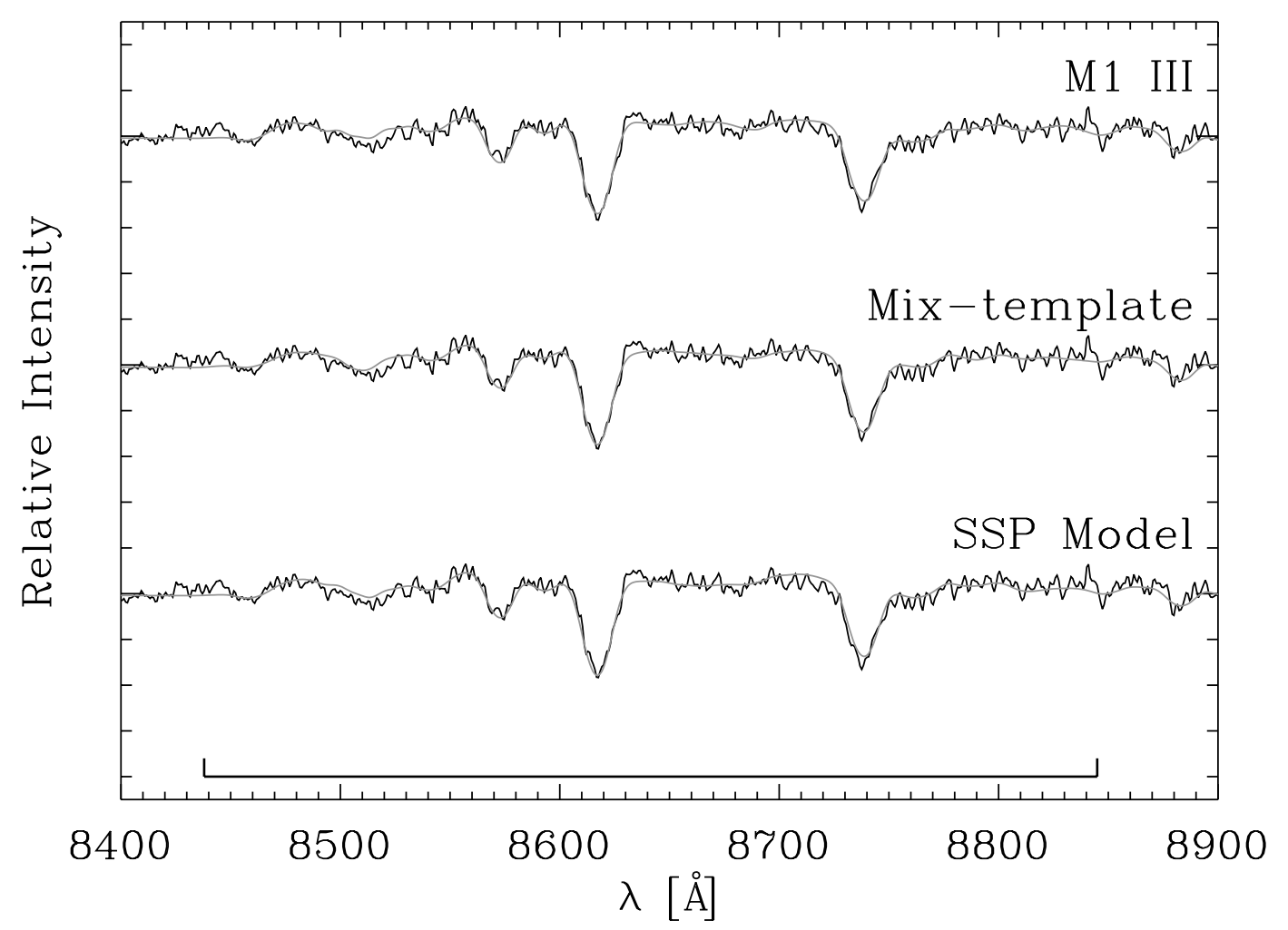

Fig. 3. Comparison between our best stellar template, mix-template, and best SSP model fits, for the continuum subtracted and low-order filtered, binned central spectrum of NGC 5326. The fitting region has been indicated with a line at the bottom of the figure In this case the best fitting template is the SSP model.

spectral types and combined stellar template. The patterns in this figure are representative of those found for all the galaxies in the sample. There is a group of stellar templates that do significantly better than the others; in particular, the A2, F2 and M4 templates consistently yield poor fits in all the galaxies. For the SSP models, the $\chi^{2}$ distributions are fairly flat as a function of age for a given metallicity, except for ages below $\sim 2$ Gyr, where the quality of the fits quickly degrades. This behavior is expected since, at younger ages, the Ca II equivalent widths significantly decrease and the Paschen series become noticeable (see Vazdekis et al. 2003). At each age, the fits are sensitive to metallicity.

For the spectrum used in Fig. 2, the best fit is obtained with a SSP model corresponding to $[\mathrm{Fe} / \mathrm{H}]=+0.2$ and $3.16 \mathrm{Gyr}$. It is worth noting that this SSP model should not necessarily be considered as the best fit to the stellar populations of this galaxy (see below). For the entire sample, the $\chi^{2}$ for the best SSP model, best stellar template and mix-template are quite similar, and for 16 of the 19 objects the SSP templates provide marginally better fits. This result demonstrates that synthetic SSP spectra, convolved to the resolution of the data, can provide accurate templates for kinematic analysis of galaxy spectra. The high quality of the SSP fits is most-likely due to their ability to minimize the template mismatch problem. While the stellar populations of bulges are likely to contain a mixture of ages and a range of metallicities, the SSP models provide a better approximation to galaxy spectra than a single stellar spectrum. Our results indicate that late KIII stars and early $\mathrm{M}$ stars provide a better approximation to the integrated light of bulges than A, F, and G stars in agreement with Vazdekis et al. (2003). Table 1, Col. 13, lists the overall best template for each galaxy. Note that, for three galaxies (NGC 5389, NGC 5719, NGC 5746), the M1III and K3I stellar templates provide the overall best fit to the galaxy spectra.

In Fig. 3 we plot the central spectrum of NGC 5326 and the best-fitting stellar (M1 III), mix and SSP templates, convolved with their respective kinematic solutions. The SSP model represents a very good fit for the whole sample of galaxies spectrum. Fitted output parameters for the best template stars (G8III, K2III, K3I, K3III, K5III, K6V, M1III), mix-template and best SSP model are shown in Fig. 4 for the entire profile of NGC 5326. The results show an excellent agreement. Therefore we adopt the solution from our best SSP template for each galaxy to obtain kinematic profiles.

A point to remark is that no inferences on the stellar population age and metallicity of the galaxies are presented here from the best-fitting SSP models. The reason is that an important relation has been shown to exist between the continuum shape and the stellar populations in this spectral region (Cenarro et al. 2003; Vazdekis et al. 2003). Since we remove this continuum, as required by FOURFIT, we neglect its valuable information for constraining galaxy stellar populations. Therefore the most useful information left in our spectra is the equivalent width of the Ca II feature, which does not vary much for SSPs of the age and metallicity regimes considered here. The wavelength region that we have used to derive the kinematics excludes the strong TiO molecular bands seen in this spectral range. Therefore we are left with an almost 


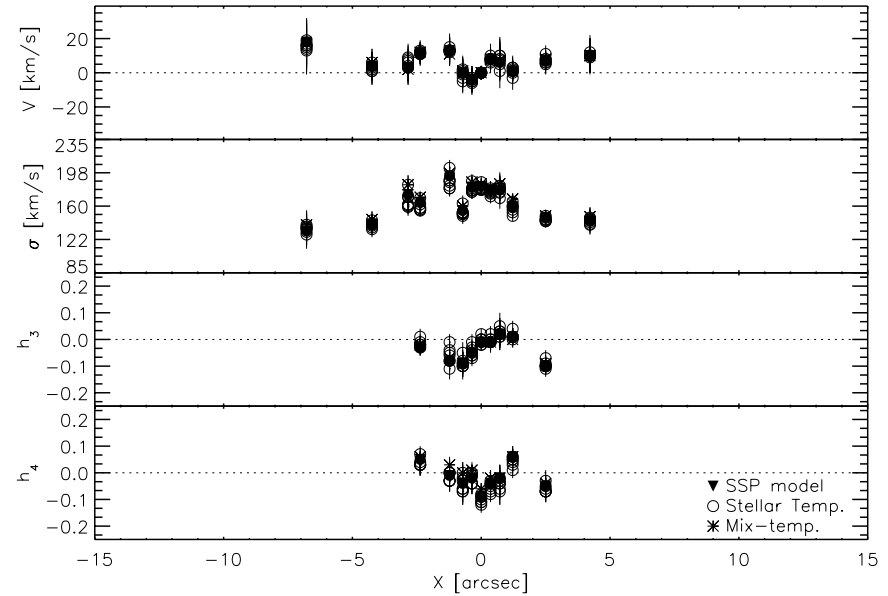

Fig. 4. Comparison of the profiles obtained with our best stellar templates (G8III, K2III, K3I, K3III, K5III, K6V, M1III), mix-template and best SSP model fit of NGC 5326.

featureless continuum shape which is straightforward to subtract. A different methodology is required to be able to analyze their stellar populations on the basis of the SSP models. Methods for extracting useful information from the full SSP spectra are discussed in Vazdekis (1999) and Vazdekis \& Arimoto (1999). The stellar population analysis of this galaxy sample will be presented in a forthcoming paper (FalcónBarroso et al. 2003).

The use of SSP models has a potential to minimize the template mismatch problems that have traditionally affected galaxy kinematic measurements. Whereas the KIII stellar templates have been shown to provide reasonably good fits to elliptical galaxy spectra in the optical region, one expects that the SSP models should significantly improve the kinematical fits. The blue and visible have been proven to have large sensitivities to the age, metallicity and abundance ratios (Rose et al. 1994; Worthey et al. 1994; Vazdekis et al. 1996; Vazdekis 1999). Our results suggest that the requirement of observing template stars with the same instrumental setting as the galaxies is not compelling. The use of SSP models as templates allows us to use all of the available observing time for the targets, without the need to take spectra of template stars. An important application of SSP models should be in the field of highredshift galaxy kinematics, where the measurement of the same spectral range in stars and target galaxies would require changing the instrumental setup, and where the redshift of the target objects is not generally known a priori.

The main limitation of the use of SSP models is that it relies on a well designed stellar library to produce the full stellar population synthesis. However, the method is expandable to include combinations of SSP models to account for composite populations. Potential applications are the study of age differences between bulge and disk, or population signatures of kinematically-decoupled cores.

\subsection{Errors and simulations}

Although FOURFIT gives the formal errors of the fit, we have performed simulations to establish the level of confidence the
Table 3. Errors from simulations.

\begin{tabular}{|c|c|c|c|c|c|c|c|c|}
\hline Param. & \multicolumn{2}{|c|}{$V\left[\mathrm{~km} \mathrm{~s}^{-1}\right]$} & \multicolumn{2}{|c|}{$\sigma\left[\mathrm{km} \mathrm{s}^{-1}\right]$} & \multicolumn{2}{|c|}{$h_{3}$} & \multicolumn{2}{|c|}{$h_{4}$} \\
\hline$S / N$ & 20 & 33 & 20 & 33 & 20 & 33 & 20 & 33 \\
\hline std(SSP) & 15 & 13 & 29 & 15 & - & 0.07 & - & 0.08 \\
\hline std(Stars) & 18 & 16 & 34 & 18 & - & 0.07 & - & 0.10 \\
\hline
\end{tabular}

Notes: Standard deviations for each parameter at the different signalto-noise ratios measured from the simulations (see Sect. 3.5).

code can produce on $\left(V, \sigma, h_{3}\right.$ and $\left.h_{4}\right)$ at $S / N=33 \mathrm{pix}^{-1}$, and $(V, \sigma)$ at $S / N=20 \mathrm{pix}^{-1}$.

For the simulations two different tests have been performed. In the first one we convolved an SSP model $([\mathrm{Fe} / \mathrm{H}]=0.0$, $t=5.01 \mathrm{Gyr}$ ) with a wide range of LOSVDs to generate model galaxy spectra. The LOSVDs included several velocities, velocity dispersions, $h_{3}$ and $h_{4}$ mapping a representative parameter space covered by our sample of galaxies $(V \in[800,2000]$, $\sigma \in[50,300], h_{3}$ and $\left.h_{4} \in[-0.1,0.1]\right)$. The inclusion of higher velocities (up to $4000 \mathrm{~km} \mathrm{~s}^{-1}$, to match our furthest galaxy) doesn't affect our results. Eight different samples of white noise were added to our model galaxy spectra to yield $S / N$ ratios of 20 and $33 \mathrm{pix}^{-1}$. For each one of the noise sample at the two $S / N$ levels the full battery of LOSVDs was recovered. Given the fact that all SSP models give very similar $\chi^{2}$ values, only a representative subsample of $3 \mathrm{SSP}$ models covering the models parameter space $([\mathrm{Fe} / \mathrm{H}]=-0.7, \mathrm{Age}=7.98 \mathrm{Gyr} ;[\mathrm{Fe} / \mathrm{H}]=0.0$, Age $=5 \mathrm{Gyr} ;[\mathrm{Fe} / \mathrm{H}]=0.2$, Age $=15.85 \mathrm{Gyr}$ ) was used to perform these simulations. We have simulated both Gaussian and non-Gaussian LOSVDs, however we only fitted pure Gaussians to the spectra of $S / N=20 \mathrm{pix}^{-1}$, for which we only modeled the $V$ and $\sigma$ errors due to LOSVD mismatching in the $S / N=20$ spectra. In the second test we created the galaxy spectra from a stellar template (K3I). Spectra from the K3I, K5III and M1III stars were used as kinematic templates. The choice of these templates is due to their ability to produce, overall, the lowest values of $\chi^{2}$ when fitted to the sample of galaxies. We have convolved the model galaxy spectra with the same LOSVDs and added the same white noise samples as in the first test.

The main results of the simulations are summarised in Table 3. The table gives the standard deviations of the distributions ( parameter $_{\text {out }}-$ parameter $_{\text {in }}$ ), obtained from the simulations in each test, for the full set of parameters and white noise samples. This is a measurement of the uncertainty when working at the mentioned $S / N$ ratios. We use the uncertainties obtained from the simulations with the SSP models and display them as error bars on the right-hand side of each kinematic profile, see Appendix A. In Fig. 5, the left panels show the distribution of the recovered LOSVDs compared to the input ones at each sampled value of $\sigma$ and $h_{4}$ for each test for $S / N>33$. The values shown below each histogram represent the median (within square brackets) and standard deviation of the distribution, found at those values of $\sigma$ and $h_{4}$. The right panels, on the other hand, show the total distribution and standard deviation 

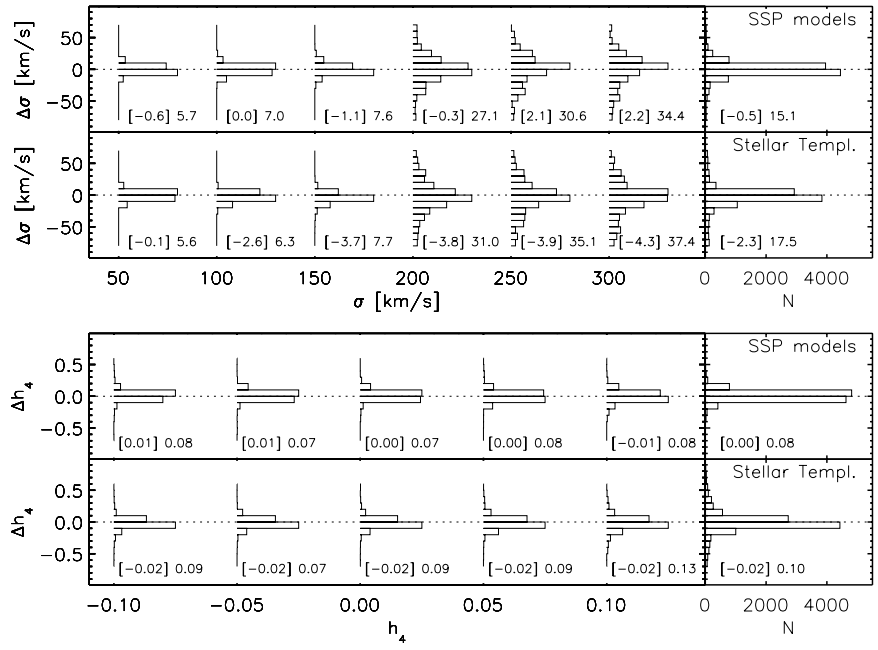

Fig. 5. Recovery of $\sigma$ and $h_{4}$ via FOURFIT using SSP models and stellar templates. The left panels show the distribution of the recovered LOSVDs compared to the input ones at each sampled value of $\sigma$ and $h_{4}$ for each test at $S / N>33$. The right panels, on the other hand, show the total distribution (i.e. the sum of the individual ones on the right panels). For each $\sigma$ and $h_{4}$ we plot a histogram of the $\Delta(\sigma)=\sigma($ out $)-\sigma($ in $)$ and $\Delta\left(h_{4}\right)=\mathrm{h}_{4}($ out $)-\mathrm{h}_{4}$ (in) obtained from the 14400 realisations corresponding to 8 white noise realizations. The values shown below each histogram represent the median (within square brackets) and standard deviation found at those values of $\sigma$ and $h_{4}$.

(i.e. the sum of the individual ones) from which we have obtain the values shown in Table 3. No significant differences are found between the distributions obtained from the SSP models and stellar templates. This result strengthens our approach of using SSP models as kinematic templates.

The simulations show two effects worth mentioning. First, there is no systematic underestimate of the velocity dispersion for low input velocity dispersions (see Fig. 5). This effect, if present, might bias our velocity dispersion results for our two smallest bulges, see Fig. 8. Such an effect has been previously reported by Joseph et al. (2001) using a different code (i.e. FCQ), finding an overestimation of $\approx 10 \mathrm{~km} \mathrm{~s}^{-1}$ at $\sigma=50 \mathrm{~km} \mathrm{~s}^{-1}$. Second, as we move towards larger velocity dispersions, the uncertainty in $\sigma$ also increases (from $6 \mathrm{~km} \mathrm{~s}^{-1}$ at $\sigma=50 \mathrm{~km} \mathrm{~s}^{-1}$, to $15 \mathrm{~km} \mathrm{~s}^{-1}$ between $\sigma=100-200 \mathrm{~km} \mathrm{~s}^{-1}$ and $32 \mathrm{~km} \mathrm{~s}^{-1}$ between $\sigma=250-300 \mathrm{~km} \mathrm{~s}^{-1}$ ). Hence, although the Gauss-Hermite expansion conforms a description of the LOSVD with a set of orthogonal functions and therefore we only expect major coupling between $\gamma$ (the peak of the LOSVD) and $\sigma$ (Larsen et al. 1983), $V$ and $h_{3}$, and $\sigma$ and $h_{4}$, we have detected other correlations when working at low signal-tonoise ratios. The expected coupling of $V$ with $h_{3}$ and $\sigma$ with $h_{4}$ appears in our simulations. Similar results have been found by authors who have performed similar tests (van der Marel \& Franx 1993; Hau et al. 1999).

We have decided to impose a $S / N=33$ as the minimum level to determine $h_{3}$ and $h_{4}$. This is a slightly lower level than that suggested by van der Marel \& Franx (1993) $\left(S / N \simeq 40 \mathrm{pix}^{-1}\right)$ to give a good estimation of the

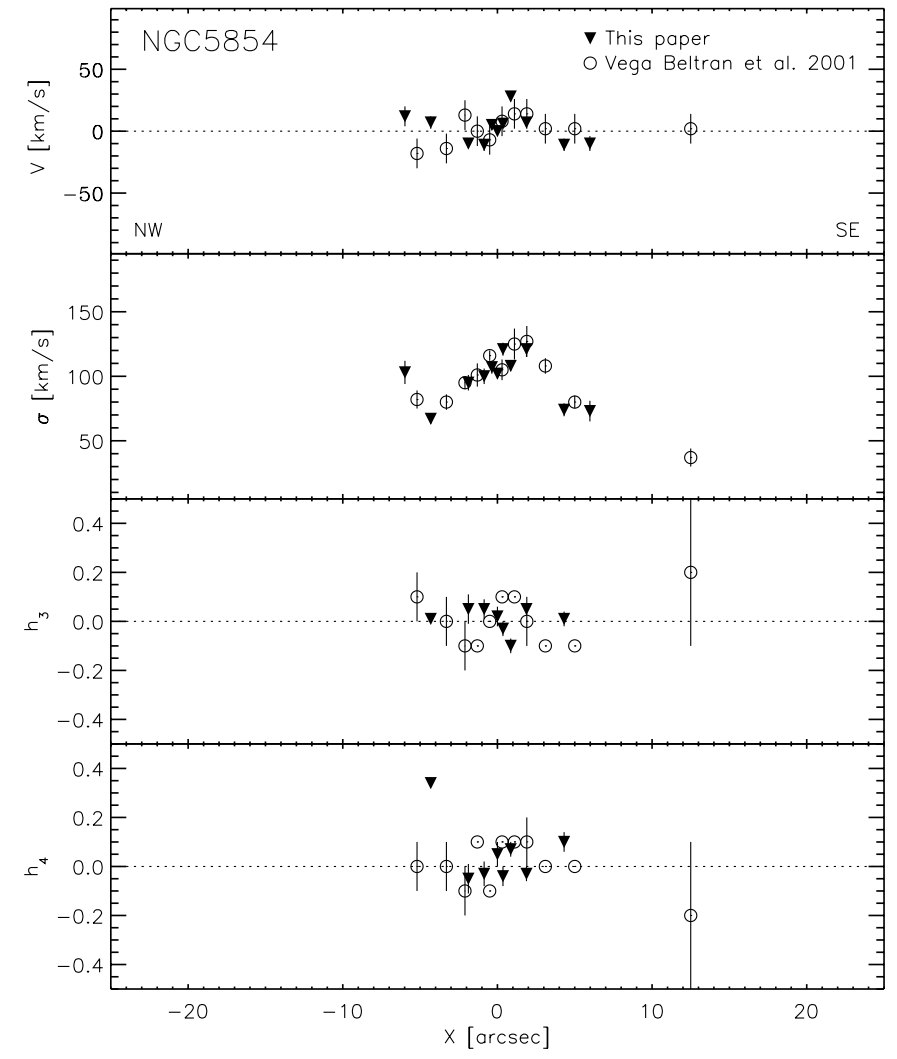

Fig. 6. Comparison between our best SSP model fit and Vega Beltrán et al. (2001) for NGC 5854.

Gauss-Hermite higher order moments. Nevertheless in most cases we obtain $S / N \geq 40$ for the profile central position (see Table 1).

\subsection{Measurement of the $\mathrm{CaT}^{*}$ index}

We have measured the recently defined Paschen-free $\mathrm{CaT}^{*}$ index (Cenarro et al. 2001a), that measures the strength of the Ca II lines corrected from the contamination by Paschen lines, from our flux calibrated spectra. We have made used of the FORTRAN routine indexfits (part of the $\mathrm{R}_{\mathrm{E}} \mathrm{D}_{\mathrm{m}}^{\mathrm{uc}} \mathrm{E}$ package), kindly made available to us by Javier Cenarro. No velocity dispersion correction and zero-point offset have been performed. Errors in the $\mathrm{CaT}^{*}$ index include contributions from radial velocity uncertainties and from $S / N$. The first one is obtained by the $\mathrm{CaT}^{*}$ index from 100 random simulations within the velocity range $[V-\Delta V, V+\Delta V]$. The latter is determined using Eq. (A37) from Cenarro et al. (2001a). Both terms are added in quadrature. The uncertainty from the $S / N$ dominates the $\mathrm{CaT}^{*}$ error.

\subsection{Comparison with the literature}

We have compared our best SSP model fits with those in the literature. Unfortunately, published minor axis kinematic profiles are scarce. We present the comparison in Figs. 6 and 7 for NGC 5854 and NGC 7332 respectively. An excellent agreement is found for the two galaxies, with no major systematic deviations, supporting our approach of using synthetic 


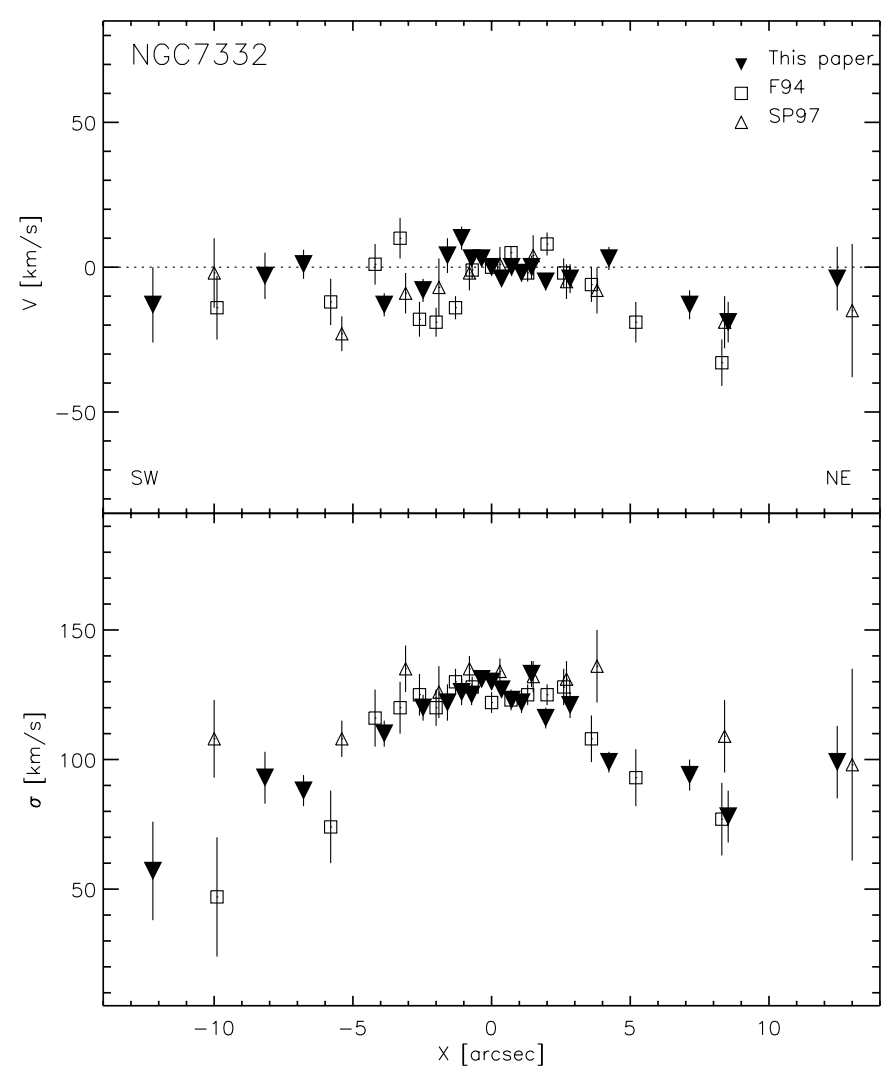

Fig. 7. Comparison between our best SSP model fit with Fisher et al. (1994) (F94) and Simien \& Prugniel (1997) (SP97) for NGC 7332.

SSP models. This agreement is particularly good for the velocity dispersion of NGC 5854, where our results follow the same trend obtained by Vega Beltrán et al. (2001). The comparison for NGC 7332 reveals also good agreement with Fisher et al. (1994) and Simien \& Prugniel (1997).

\section{Results}

Kinematic profiles for the 19 galaxies in the sample are shown in Fig. A.1 (Appendix A). For each galaxy we show profiles of mean heliocentric velocity $V$, velocity dispersion $(\sigma), h_{3}$ and $h_{4}$ Gauss-Hermite coefficients, and Ca II triplet index $\mathrm{CaT}^{*}$, along the galaxy's minor axis. Abscissae are centered on the brightness peak of the long-slit spectrum, i.e. on the photometric center of the galaxy since we don't expect large centering errors. Negative abscissae correspond to the minor axis that projects away from the disk (the "dust-free" side), whose position angle on the sky is given at the top next to the galaxy's name. Circles are measurements derived from $S / N>33 \mathrm{pix}^{-1}$ spectra, and triangles are $V, \sigma$ and $\mathrm{CaT}^{*}$ derived from $20<S / N<33$ spectra. Error bars in each point are the formal error estimates coming from FOURFIT code. Measurement uncertainties derived from the simulations described in Sect. 3.5 are shown with vertical bars on the right edge of each plot for the $S / N>33$ spectra and, for $V$ and $\sigma$, for $20<S / N<33$ as well (longest bar). The contour plot draws isophotes of the HST NICMOS F160W images (Peletier et al. 1999), drawn to scale. The length of the 1.2 arcsec wide slit delimits the range along the minor axis profile from which spectra has been binned to obtain a

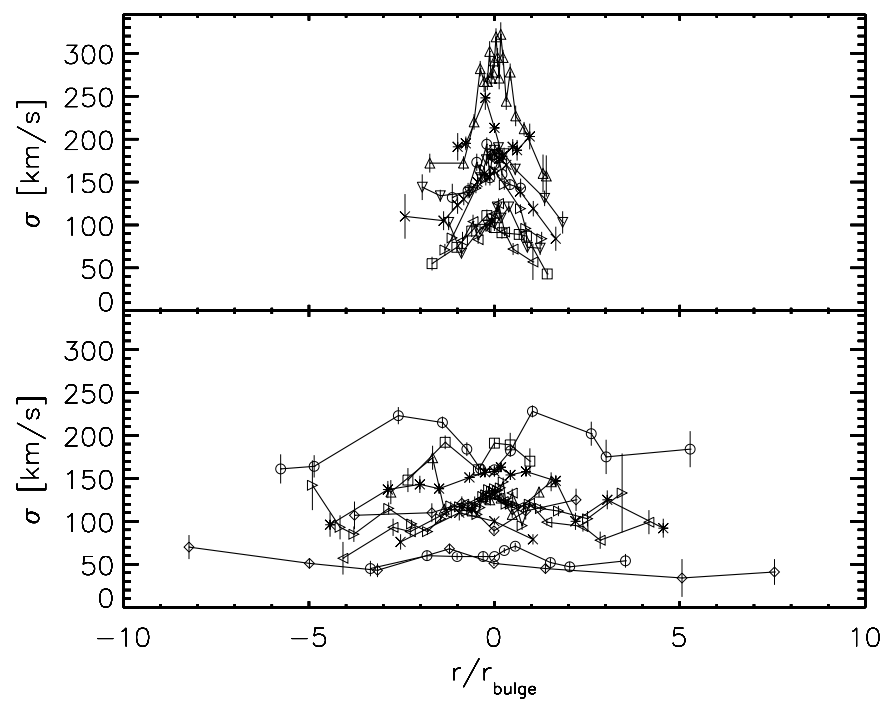

Fig. 8. Velocity dispersion profiles for our sample of galaxies. Bulges with centrally-peaked velocity dispersions profiles are drawn in the top panel, whereas bulges with flat velocity dispersion profiles are plotted in the bottom panel. In the abscissa, radii have been normalized to the minor axis bulge radius (Table 1, Col. 12), defined as the radius along the minor axis where bulge and disk have equal surface brightness.

$S / N>20$. The high spatial resolution of the NICMOS images $(0.075 \mathrm{arcsec} / \mathrm{pixel}, P S F=0.18 \operatorname{arcsec} F W H M)$ provides a clue on the level of substructure existing within the spectroscopic slit. Due to the lack of a NICMOS image for NGC 7332, a WFPC1 image, retrieved from the HST archive, has been used instead. Vertical dotted lines indicate the minor axis bulge radius and center of the galaxy (see Table 1 for more details).

Owing to the short integration times and the $S / N$ requirements for kinematic extractions derived from the simulations (Sect. 3.5), the kinematic profiles typically extend to 510 arcsec from the nucleus only. Only for the large galaxy NGC 7331 do kinematic profiles extend over 20 arcsec on the dust-free side. All profiles are drawn to the same horizontal scale. In the following subsections we briefly address the global behavior of each kinematic parameter.

\subsection{Velocity profiles}

As expected for the minor-axis alignment of the slits, mean velocity profiles are fairly flat. However, the profiles are not entirely featureless. We note three types of features.

\subsubsection{Rotation in the outer parts}

We detect non-zero rotation in the outer parts of the profiles (opposite mean velocities at both sides of the center, at the $2-\sigma$ level) in 6 out of the 19 galaxies (NGC 5389, 5689, 5746, $5838,5854,7457)$. Typical rotation amplitudes are in the range $10<V<20 \mathrm{~km} \mathrm{~s}^{-1}$. Such estimates of velocity amplitude and frequency of occurrence are highly dependent on the depth of the data. For two galaxies (NGC 5475, 5587) the profiles do 
not reach 5 arcsec and contain no information on rotation at large radii. Brighter galaxies in which the spectra extend farthest from the center show the highest $V$ at large radii. In most of these cases, the outer velocity measurements sample disk or bulge-disk transition regions. The non-zero velocities indicate that our spectroscopic slits were not perfectly aligned with the line of nodes. The slit was placed orthogonal to the major axis of the outer isophotes. Hence the misalignment might reflect an isophote twist in the outer parts sampled by the spectra, due e.g. to warps or spiral arms, or a distortion of the inner disk velocity field due to a bar or a spiral arm. Note that a misalignment of $5^{\circ}$ or more could easily explain such amplitudes in velocity.

\subsubsection{Inner rotation}

We detect inner minor-axis rotation in 8 galaxies (NGC 5443, $5587,5689,5707,5838,5965,6010,7457)$ and possibly also in NGC 5389, 5422, 5854. Whenever the profiles extend beyond the nuclear region, inner rotation is delimited by a change in the velocity profile slope. These bulges thus contain kinematically distinct regions (hereafter KDR). We do not intend to set a link between these KDR and counterrotating or otherwise kinematically decoupled cores in ellipticals and bulges; full counterrotation cannot be established from our minor-axis kinematic profiles. Rather, we associate the velocity features to slight variations in the kinematic structure, related to inner components such as disks or bars.

Theoretically the simplest way to explain a minor-axis rotation in a bulge is to assume we are dealing with a triaxial object. In a triaxial object the position angle of the apparent minor axis can be different from that of the projected intrinsic short axis of the object (de Zeeuw \& Franx 1991). Moreover, the photometric and kinematic minor axes may be misaligned as a result of the wide range of possible locations of the total angular momentum in a triaxial object (Binney 1985). In practice, whether minor-axis rotation traces a triaxial bulge can only be checked using integral field spectrographs e.g. SAURON (Bacon et al. 2001), or measuring kinematics along different positions angles of the galaxy (Statler \& Smecker-Hane 1999).

The typical minor-axis radial extent of the KDR is 2-3 arcsec, or $0.5 \mathrm{kpc}$ at the median distance for the sample. In all of the eight galaxies listed above as KDR, isophotal analysis of the HST/NICMOS F160W images (our work, unpublished) reveals an inflection in the $C_{4}$ isophotal coefficient of the $\cos (4 \theta)$ term that measures disky deviations of the isophotes from pure ellipses (e.g. Jørgensen et al. 1992). $C_{4}$ amplitudes of the features are above $1 \%$ in all cases. This suggests the presence of flattened subcomponents in the inner regions of the bulges showing up as excesses in the bulge surface brightness profiles (Balcells et al. 2003). One probably sees a mixture of inner disks and inner bars. In four of the eight cases the isophotal analysis reveals changes in isophote position angle $\left(\triangle \mathrm{PA} \geq 10^{\circ}\right)$ at the same radius, which are likely to correspond to inner bars but could also trace misaligned disks. The small rotation amplitudes and the lack of isophotes elongated perpendicularly to the disks suggests that none of our bulges show the type of orthogonal bulge kinematics found in NGC 4698
(Bertola et al. 1999) and NGC 4672 (Sarzi et al. 2000). The four remaining cases with no isophotal misalignment probably correspond to inner bars, as an aligned disk has its line of nodes parallel to the minor axis.

The most prominent nuclear KDR is that of NGC 5838. The nucleus of NGC 5838 harbors an ordered ring or disk of dust and young stars (Peletier et al. 1999), which is oriented orthogonal to the slit. The dust may be responsible for the asymmetric rotation pattern.

It is worth noting that none of the KDR have distinct colors $B-I$ and $I-H$ (Peletier et al. 1999) on the dust-free side of the galaxies, nor do they have corresponding features in the $\mathrm{CaT}^{*}$ profiles (Fig. A1). This may suggests that the velocity features are not associated to distinct stellar populations (e.g. Davies et al. 2001).

\subsubsection{Kinematically offset nuclei}

In two galaxies (NGC 5707, 7331) the velocities at large radii appear offset in the same direction relative to the nuclear velocity. NGC 5707 has a nuclear disk or bar extending over 1 arcsec, which is well aligned with the major axis of the outer disk and partially obscured by a dust lane; the dust might be responsible for the offset velocity measurement at the photometric center of the galaxy. In NGC 7331, the entire region from -4 arcsec to +4 arcsec appears displaced $15-20 \mathrm{~km} \mathrm{~s}^{-1}$ from the velocities further out. It is unclear whether dust extinction can be responsible for this structure, or whether the bulge might actually wander in the potential of the galaxy. The major-axis kinematics of this galaxy is complex, and its outer spiral structure is asymmetric; this could indicate a recent accretion event and/or the motion of the luminous material within the dark matter halo of the galaxy (Noordermeer et al. 2001). Results on the major-axis kinematics of this galaxy are puzzling. While Prada et al. (1996) reported to have found the bulge in NGC 7331 to counter-rotate, a more recent work by Bottema (1999) contradicts this result.

\subsection{Velocity dispersion profiles}

Velocity dispersion profiles trace the dynamical temperature of the bulges and are key ingredients for dynamical modeling of the bulges. They also provide diagnostics for distinguishing between bulge and disk on the basis of the internal dynamics.

For our galaxies, the minor-axis velocity dispersion profiles show a wide diversity of shapes, from flat to strongly centrally peaked. Velocity dispersion gradients, $(\Delta \sigma$ per unit minor-axis $r_{\mathrm{e}}$ ) were computed by fitting linear relations to the negative-abscissa dispersion profiles of Fig. A.1, which correspond to the dust-free side of the bulges. Minor-axis $r_{\mathrm{e}}$ were derived using the $r_{\mathrm{e}}$ values from Balcells et al. (2003), which derive from $H$-band surface brightness profiles from HST-NICMOS images, and ellipticities at the bulge effective radius $\epsilon_{\mathrm{B}}\left(r_{\mathrm{e}}\right)$ obtained from the ellipticity profiles in the same paper. Dispersion gradients range from 0 to $-70 \mathrm{~km} \mathrm{~s}^{-1} r_{\mathrm{e}}^{-1}$, with a median of $-21 \mathrm{~km} \mathrm{~s}^{-1} r_{\mathrm{e}}^{-1}$ and a mode of only $\sim 10 \mathrm{~km} \mathrm{~s}^{-1} r_{\mathrm{e}}^{-1}$. 


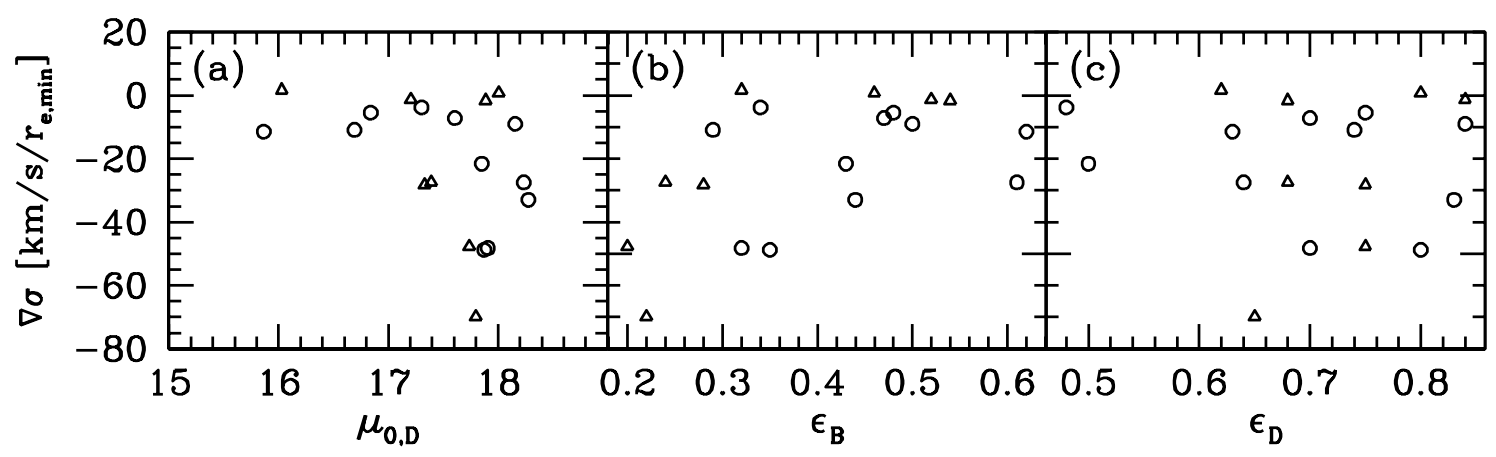

Fig. 9. Dependency of the velocity dispersion gradient on galaxy parameters. a) $\nabla \sigma$ against face-on $H$-band disk central surface brightness $\mu_{0, \mathrm{D}}$. b) $\nabla \sigma$ against bulge ellipticity $\epsilon_{\mathrm{B}}$. c) $\nabla \sigma$ against disk ellipticity $\epsilon_{\mathrm{D}}$. Barred or box-peanut shaped galaxies in our sample are represented with open triangles.

Figure 8 displays all the velocity dispersion profiles, presented on two panels for clarity; the top/bottom panel shows the half sample with highest/lowest $\nabla(\sigma)$. In order to show whether the profiles sample only the bulge or extend to the disk region, we normalize each abscissa to $r_{\text {bulge }}$, the minor-axis radius where bulge and disk contribute equally to the surface brightness profile (listed in Col. 12 of Table 1). Most profiles extend well beyond $r / r_{\text {bulge }}=1$, indicating that the profiles reach to the region dominated by disk light according to the bulge-disk decomposition.

Three effects may influence the shape of the minor axis velocity dispersion profiles in our galaxies, (i) the intrinsic velocity structure of the bulge, (ii) the galaxy inclination, which determines which projection of the velocity ellipsoid is sampled by the data, and (iii) the brightness of the disk in the region sampled by the kinematic profiles.

Because most profiles reach the region where the surface brightness is dominated by the disk (Fig. 8), flat dispersion profiles imply similar dispersions for bulge and host disk. At the median inclination of the sample, $i=73^{\circ}$, minor-axis dispersion profiles preferentially sample the radial component of the velocity dispersion ellipsoid. Our flat profiles then indicate a surprisingly high radial velocity dispersions in the regions dominated by disk light, and an equally surprising continuity of the radial velocity dispersions into the region of the bulge. Steep dispersion profiles, in contrast, indicate distinct radial dispersions in the bulge and host disk, with dispersions increasing toward the center as is expected in dynamically hot, self-gravitating ellipsoids.

The disk central surface brightness influences the velocity dispersion gradient. Figure 9a shows the distribution of $\nabla(\sigma)$ against the face-on disk central surface brightness $\mu_{0}$. Bright disks have shallow dispersion profiles, while the steepest profiles are found in faint disks. We find it unlikely that this dependency arises from biases in the derivation of the kinematic profiles. Our simulations (Sect. 3.5) show that dispersions as low as $\sigma \sim 50 \mathrm{~km} \mathrm{~s}^{-1}$ are well reproduced by the FOURFIT algorithm. Selection biases would hamper the detection of disk dispersions for faint disks first, but dispersion gradients of all values are found for these disks. The lack of steep dispersion profiles in bright disks appears therefore real.
Figure $9 \mathrm{~b}$ shows the distribution of $\nabla(\sigma)$ against $\epsilon_{\mathrm{B}}\left(r_{\mathrm{e}}\right)$, the ellipticity measured at the bulge effective radius. A trend is found, in the sense that more flattened bulges show shallower dispersion profiles. This dependence does not arise from viewing-angle effects: Fig. 9c shows that $\nabla(\sigma)$ does not depend on the galaxy inclination. Shallow-profile objects may be kinematically described as thickened disks. In low-ellipticity bulges, the $\sigma$ profiles increase toward the center, again as expected from hot ellipsoids.

Kormendy (1993) discusses that some bulges share strong similarities with disks, on the basis of their low velocity dispersions, high $V_{\max } / \sigma_{0}$ for their ellipticity, and nuclear spiral structure. The disky nature of bulges is usually discussed in relation to late-type, low-mass bulges (Kormendy 1993). Our dispersion profiles indicate that this situation affects also earlytypes, including several S0's.

It appears that the disky nature of bulges cannot be established on the basis of spheroid luminosity; our velocity dispersion gradients do not correlate with bulge luminosity or with central velocity dispersion. Gradients do not correlate either with morphological type index, bulge Sérsic index $n$, bulge and disk scale lengths and bulge effective surface brightness. We have searched for a dependency on the presence of bars or boxpeanut shaped bulge isophotes. Such galaxies are depicted with triangles in Fig. 9. While bar detection is difficult at the inclinations of our sample galaxies, it is apparent that barred and box-peanut bulges do not occupy preferred regions in Fig. 9.

The massive bulge of NGC 5746 (Sb) is the only object showing a strongly-defined central velocity dispersion minimum. A weak central minimum is also found in NGC 5443. Central velocity dispersion minima are generally understood as tracing embedded disks, bars, or low-mass spheroids. Therefore, given the abundance of isophotal substructure in the nuclei of these galaxies, it is surprising that central velocity dispersion minima are so infrequent among our objects. As mentioned above, our sample includes six box- or peanut-shaped bulges. For these objects, the bar producing the peanut may not reach the center; or, alternatively, the central dispersion may be affected by the presence of secondary nuclear stellar bars/disks, with dispersion minima in bars being found for given viewing angles only. In NGC 5746 the local central minimum may be 
due to the main bar reaching all the way to the inner arcsecond, or to the presence of a secondary bar or nuclear disk.

\subsection{High-order Gauss-Hermite terms}

Profiles of the $h_{3}$ skewness term of the LOSVD are consistent with zero in most cases. In the few cases with significant non-zero $h_{3}$ (NGC 5475, 6010, 7331), $h_{3}$ does not have an opposite sign to the mean velocity that is typical of major axis profiles. This is reasonable as any rotation detected in the spectra is due to slight slit misalignment respect to the minor axis.

Similarly, the kurtosis profiles $h_{4}$ are generally flat and close to zero. In 12 galaxies however, $h_{4}$ is significantly positive above 0.1 somewhere in the profile, denoting centrally-peaked LOSVDs.

\subsection{The $\mathrm{CaT}^{*}$ profiles}

We have measured the $\mathrm{CaT}^{*}$ profiles for our sample of galaxies in order to trace correlations between kinematic and population features along the galaxies minor axis. Our analysis of the profiles does not show any correlation with either kinematic $(V, \sigma$, $h_{3}, h_{4}$ ) or photometric parameters (from Balcells et al. 2003). The lack of correlation might be due to the very small dependence of the $\mathrm{CaT}^{*}$ on age and metallicity for the values we expect for this type of galaxies (Vazdekis et al. 2003).

In a forthcoming paper (Falcón-Barroso et al. 2003) we will present an extensive study of the near-IR Ca II triplet- $\sigma$ relation for our sample of bulges. In that paper we give measurements of the recently defined near-infrared Ca II triplet indices (CaT, $\left.\mathrm{CaT}^{*}\right)$, Paschen (PaT) and Magnesium (Mg I) indices (see Cenarro et al. 2001a) for our sample of bulges. We show that both the $\mathrm{CaT}^{*}$ and $\mathrm{CaT}$ indices decrease with central velocity dispersion $\sigma$ with small scatter. The decrease of CaT and $\mathrm{CaT}^{*}$ with $\sigma$ contrasts with the well-known increase of another $\alpha$-element index, $\mathrm{Mg}_{2}$, with $\sigma$.

\section{Conclusions}

We have extracted minor-axis kinematic profiles from spectra in the Ca II near-IR region for a well-studied sample of early - to intermediate-type, inclined galaxies from the Balcells \& Peletier (1994) sample. SSP models from Vazdekis et al. (2003) at $1.5 \AA$ resolution have been used in addition to the traditional template stars. We have compared results obtained with the different templates and determined that synthetic models produce slightly better results than those from stellar templates, in the Ca II triplet region. We discuss the advantages/disadvantages of using SSP models instead of stellar templates. Our main conclusion is that synthetic models, when coming from a well constructed stellar library, help to reduce several drawbacks such as the template mismatch problem affecting all the traditional kinematical methods. We find a good agreement with high-quality kinematic profiles from the literature. SSP model spectra used as templates have clear applications for kinematic studies of galaxies at high redshift.

We find minor axis rotation in almost half of our galaxy sample. We discuss two types of minor axis rotation: rotation in the outer parts, probably due to slit misalignments, and inner rotation, which are associated to disky deviations of the isophotes from pure ellipses. We also find some cases of kinematically offset nuclei (NGC 5707 and NGC 7331) for which we do not find a clear explanation. We show that flattened bulges tend to show shallow velocity dispersion gradients and similar dispersions in bulge and inner disk, possibly indicating a thickened disk structure for these bulges. Finally, we find that our velocity dispersion gradients correlate with disk central surface brightness and with bulge ellipticity, but do not correlate with disk ellipticity. Gradients do not correlate either with bulge luminosity, bulge central velocity dispersion, bulge Sérsic index $n$, bulge effective surface brightness, galaxy morphological type, and disk or bulge effective radii.

Acknowledgements. JFB acknowledges the financial support from a PPARC studentship. The William Herschel Telescope is operated on the island of La Palma by the Isaac Newton Group in the Spanish Observatorio del Roque de los Muchachos of the Instituto de Astrofísica de Canarias. We acknowledge the professional help of the observatory staff in the operation of the WHT and the ISIS spectrograph.

\section{Appendix A: Profiles}

In this section we present kinematic profiles for each galaxy (heliocentric velocity, velocity dispersion, $h_{3}, h_{4}$ ), together with an $H$-band NICMOS (F160W) contour map from Peletier et al. (1999) (except for NGC 7332 for which a WFPC1 image, taken from the archive, is plotted instead). The length of the 1.2 arcsec wide slit delimits the range along the minor axis profile from which spectra has been binned to obtain a $S / N>20$. The $\mathrm{CaT}^{*}$ index (see Sect. 3.6) is shown in the bottom panel. Filled circles represent data points with signal to noise ratio above 33, whereas filled triangles represent data points with signal to noise between 20 and 33 . Contour levels have $0.5 \mathrm{mag}$ difference. The left hand side of the profiles, for all galaxies, corresponds the dust-free side, while the right hand side corresponds to the dusty side. The PA (on top of the figure) is the position angle (N-E) of the dust-free minor axis. Error bars determined in the simulation for each $S / N$ (see Sect. 3.5) are located on the right hand side of each panel, $S / N=33$ (short bar) and $S / N=20$ (long bar). A dotted line has been drawn, on each panel, at the level of the central value for the velocity, velocity dispersion and $\mathrm{CaT}^{*}$ index and it is at the zero value for the $h_{3}$ and $h_{4}$ parameters. Vertical dotted lines indicate the minor axis bulge radius and center of the galaxy (see Table 1 for more details). 

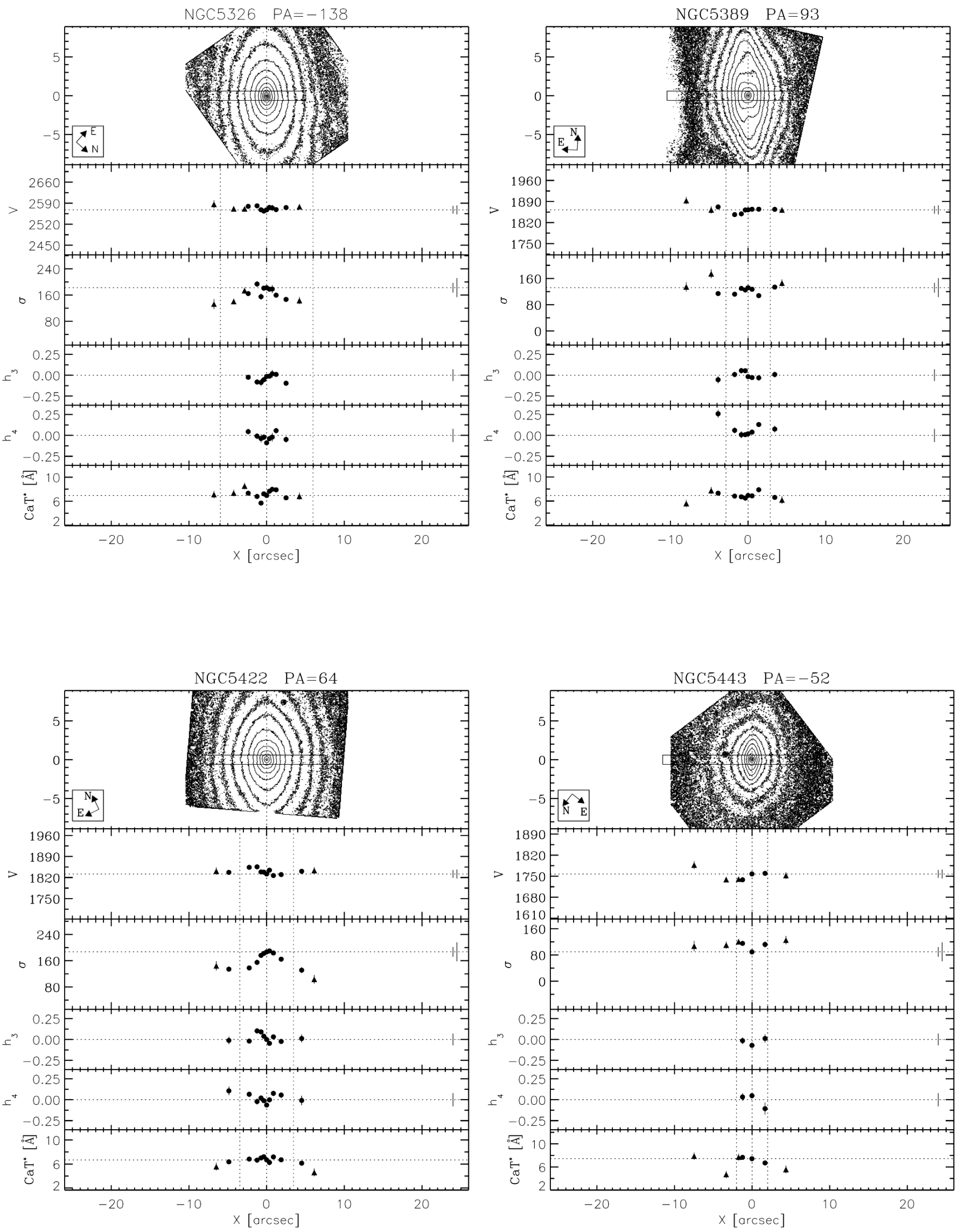

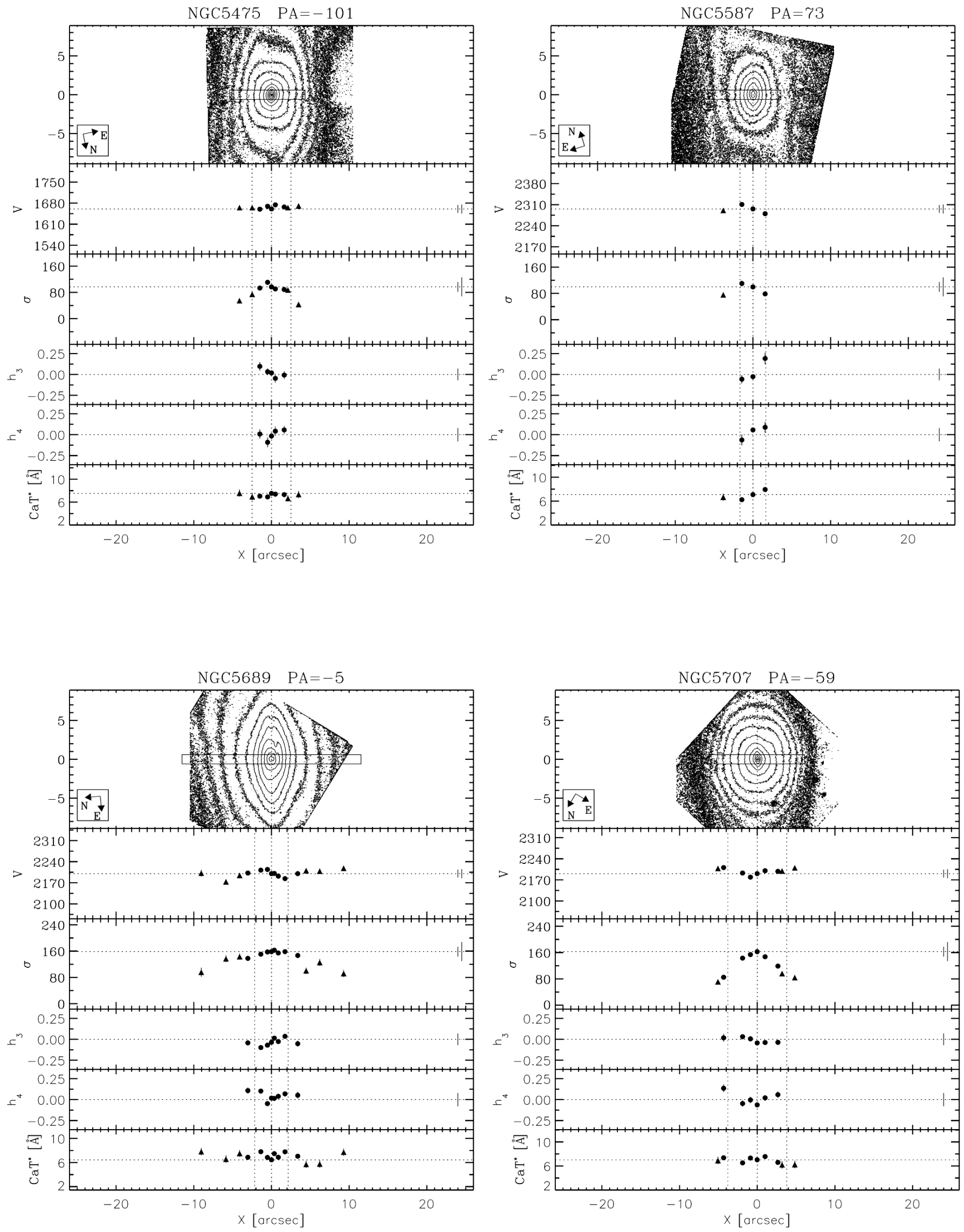

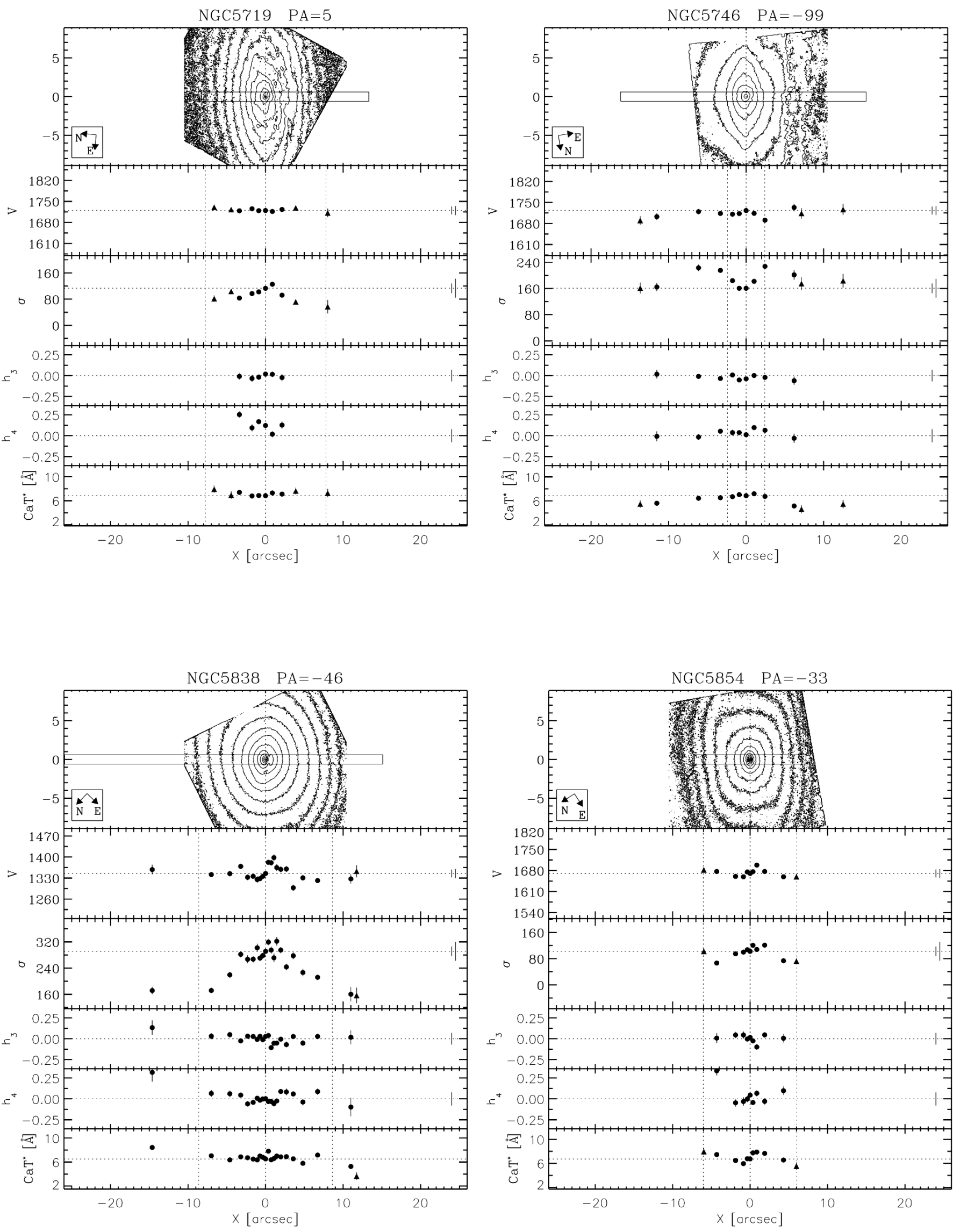

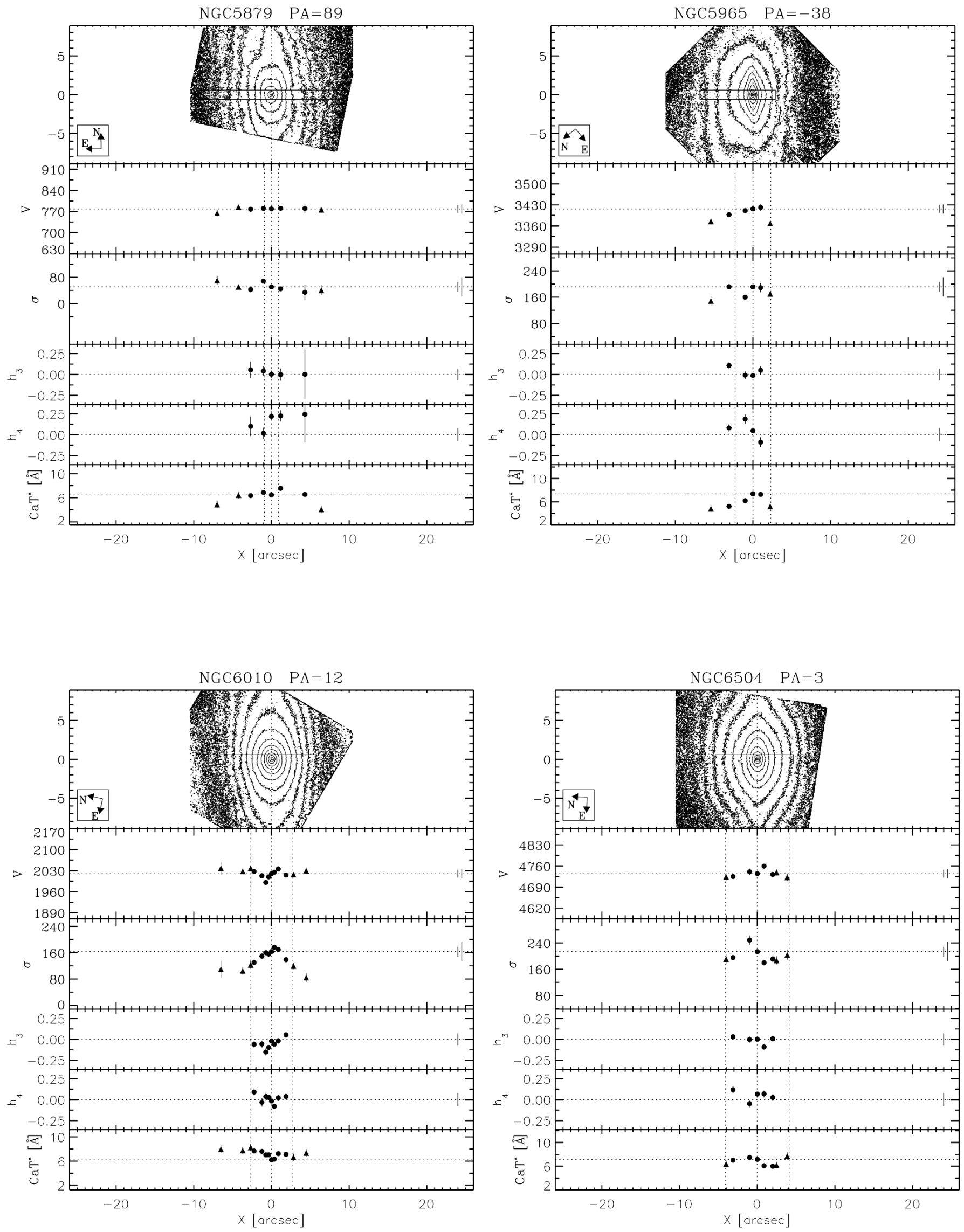

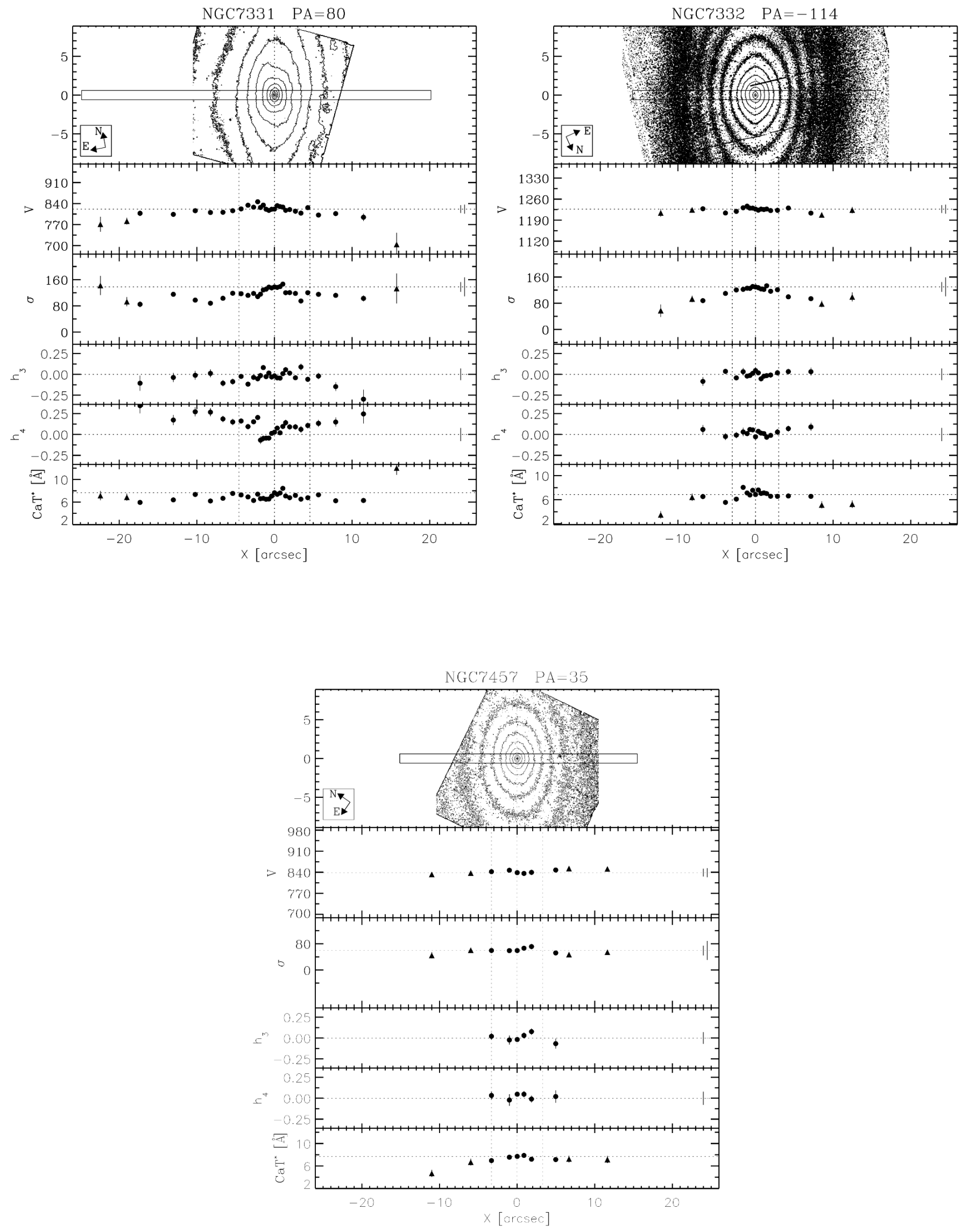


\section{References}

Bacon, R., Copin, Y., Monnet, G., et al. 2001, MNRAS, 326, 23 Balcells, M., Graham, A. W., Domínguez-Palmero, L., \& Peletier, R. F. 2003, ApJ, 582, L79

Balcells, M., \& Peletier, R. F. 1994, AJ, 107, 135

Bender, R. 1990, A\&A, 229, 441

Bender, R., Saglia, R. P., \& Gerhard, O. E. 1994, MNRAS, 269, 785

Bertola, F., Corsini, E. M., Vega Beltrán, J. C., et al. 1999, ApJ, 519, L127

Binney, J. 1985, MNRAS, 212, 767

Bottema, R. 1999, A\&A, 348, 77

Bruzual A., G., \& Charlot, S. 1993, ApJ, 405, 538

Burbidge, E. M., \& Burbidge, G. R. 1968, ApJ, 154, 857

Buzzoni, A. 1995, ApJS, 98, 69

Cannon, A. J., \& Pickering, E. C. 1993, VizieR Online Data Catalog, 3135, 0

Cardiel, N. 1999, Ph.D. Thesis, Universidad Complutense de Madrid Cenarro, A. J., Cardiel, N., Gorgas, J., et al. 2001a, MNRAS, 326, 959 Cenarro, A. J., Gorgas, J., Cardiel, N., et al. 2001b, MNRAS, 326, 981 Cenarro, A. J., Gorgas, J., Vazdekis, A., Cardiel, N., \& Peletier, R. F. 2003, MNRAS, 339, L12

Davies, R. L., Kuntschner, H., Emsellem, E., et al. 2001, ApJ, 548, L33

de Vaucouleurs, G., de Vaucouleurs, A., Corwin, H. G., et al. 1991, S\&T, 82, 621

de Zeeuw, T., \& Franx, M. 1991, ARA\&A, 29, 239

Falcón-Barroso, J., Peletier, R. F., \& Balcells, M. 2002, MNRAS, 335, 741

Falcón-Barroso, J., Peletier, R. F., Vazdekis, A., \& Balcells, M. 2003, ApJ, 588, L17

Fisher, D. 1997, AJ, 113, 950

Fisher, D., Illingworth, G., \& Franx, M. 1994, AJ, 107, 160

González, J. J. 1993, Ph.D. Thesis. University of California Santa Cruz

Hau, G. K. T., Carter, D., \& Balcells, M. 1999, MNRAS, 306, 437

Hoffleit, D., \& Warren, W. H. 1995, VizieR Online Data Catalog, 5050, 0

Jørgensen, I., Franx, M., \& Kjaergaard, P. 1992, A\&AS, 95, 489

Joseph, C. L., Merritt, D., Olling, R., et al. 2001, ApJ, 550, 668
Karachentsev, I. D., \& Makarov, D. A. 1996, AJ, 111, 794

Kent, S. M. 1984, ApJS, 56, 105

Kormendy, J. 1993, in Galactic Bulges, IAU Symp., 153, 209

Kuijken, K., \& Merrifield, M. R. 1993, MNRAS, 264, 712

Kukarkin, B. V., \& Kholopov, P. N. 1982, New catalogue of suspected variable stars (Moscow: Publication Office Nauka)

Larsen, N., Norgaard-Nielsen, H. U., Kjaergaard, P., \& Dickens, R. J. 1983, A\&A, 117, 257

Nilson, P. 1995, VizieR Online Data Catalog, 7026, 0

Noordermeer, E., Sparke, L. S., \& Levine, S. E. 2001, MNRAS, 328, 1064

Peletier, R. F., \& Balcells, M. 1997, New Astron., 1, 349

Peletier, R. F., Balcells, M., Davies, R. L., et al. 1999, MNRAS, 310, 703

Pickles, A. J. 1985, ApJ, 296, 340

Prada, F., Gutierrez, C. M., Peletier, R. F., \& McKeith, C. D. 1996, ApJ, 463, L9

Rix, H., \& White, S. D. M. 1992, MNRAS, 254, 389

Rose, J. A., Bower, R. G., Caldwell, N., et al. 1994, AJ, 108, 2054

Sargent, W. L. W., Schechter, P. L., Boksenberg, A., \& Shortridge, K. 1977, ApJ, 212, 326

Sarzi, M., Corsini, E. M., Pizzella, A., et al. 2000, A\&A, 360, 439

Simien, F., \& Prugniel, P. 1997, A\&AS, 126, 15

Statler, T. S., \& Smecker-Hane, T. 1999, AJ, 117, 839

Statler, T. S., Smecker-Hane, T., \& Cecil, G. N. 1996, AJ, 111, 1512

Tantalo, R., Chiosi, C., Bressan, A., \& Fagotto, F. 1996, A\&A, 311, 361

Tonry, J., \& Davis, M. 1979, AJ, 84, 1511

van der Marel, R. P., \& Franx, M. 1993, ApJ, 407, 525

Vazdekis, A. 1999, ApJ, 513, 224

Vazdekis, A., \& Arimoto, N. 1999, ApJ, 525, 144

Vazdekis, A., Casuso, E., Peletier, R. F., \& Beckman, J. E. 1996, ApJS, 106, 307

Vazdekis, A., Cenarro, A. J., Gorgas, J., Cardiel, N., \& Peletier, R. F. 2003, MNRAS, 340, 1317

Vega Beltrán, J. C., Pizzella, A., Corsini, E. M., et al. 2001, A\&A, 374, 394

Worthey, G., Faber, S. M., Gonzalez, J. J., \& Burstein, D. 1994, ApJS, 94,687 


\section{Online Material}




\section{Tables}

In this appendix we tabulate for each galaxy the heliocentric velocities, velocity dispersions and their errors are expressed in $\mathrm{km} / \mathrm{s}$ whereas the values for the $\mathrm{CaT}^{*}$ index, and their uncertainties, are given in $\AA$. 
J. Falcón-Barroso et al.: Minor axis kinematics of 19 S0-Sbc bulges, Online Material p 3

Table A.1. NGC 5326

\begin{tabular}{ccccccccccc}
\hline \hline $\mathrm{x}\left[^{\prime \prime}\right]$ & $\mathrm{V}$ & $\Delta V$ & $\sigma$ & $\Delta \sigma$ & $\mathrm{h}_{3}$ & $\Delta \mathrm{h}_{3}$ & $\mathrm{~h}_{4}$ & $\Delta \mathrm{h}_{4}$ & $\mathrm{CaT}^{*}$ & $\Delta \mathrm{CaT}^{*}$ \\
\hline-6.78 & 2586 & 13 & 132 & 16 & 0.00 & 0.00 & 0.00 & 0.00 & 7.11 & 0.62 \\
-4.24 & 2572 & 8 & 140 & 9 & 0.00 & 0.00 & 0.00 & 0.00 & 7.36 & 0.58 \\
-2.84 & 2571 & 8 & 173 & 10 & 0.00 & 0.00 & 0.00 & 0.00 & 8.53 & 0.54 \\
-2.37 & 2579 & 6 & 164 & 6 & -0.03 & 0.03 & 0.05 & 0.03 & 7.34 & 0.37 \\
-1.23 & 2581 & 8 & 194 & 8 & -0.08 & 0.03 & -0.01 & 0.04 & 6.79 & 0.37 \\
-0.72 & 2568 & 8 & 155 & 9 & -0.09 & 0.04 & -0.04 & 0.05 & 5.70 & 0.36 \\
-0.36 & 2564 & 7 & 181 & 8 & -0.05 & 0.03 & -0.02 & 0.03 & 7.24 & 0.26 \\
0.00 & 2568 & 4 & 182 & 5 & -0.01 & 0.02 & -0.09 & 0.02 & 6.95 & 0.24 \\
0.36 & 2576 & 6 & 178 & 7 & -0.01 & 0.03 & -0.04 & 0.03 & 7.66 & 0.28 \\
0.72 & 2574 & 10 & 178 & 12 & 0.02 & 0.05 & -0.02 & 0.05 & 7.98 & 0.37 \\
1.22 & 2569 & 6 & 159 & 6 & 0.01 & 0.03 & 0.06 & 0.03 & 7.90 & 0.37 \\
2.50 & 2575 & 5 & 147 & 6 & -0.10 & 0.03 & -0.05 & 0.04 & 6.57 & 0.37 \\
4.22 & 2578 & 10 & 143 & 11 & 0.00 & 0.00 & 0.00 & 0.00 & 6.84 & 0.61 \\
\hline \hline
\end{tabular}

Table A.2. NGC 5389

\begin{tabular}{ccccccccccc}
\hline \hline $\mathrm{x}\left[^{\prime \prime}\right]$ & $\mathrm{V}$ & $\Delta V$ & $\sigma$ & $\Delta \sigma$ & $\mathrm{h}_{3}$ & $\Delta \mathrm{h}_{3}$ & $\mathrm{~h}_{4}$ & $\Delta \mathrm{h}_{4}$ & $\mathrm{CaT}^{*}$ & $\Delta \mathrm{CaT}^{*}$ \\
\hline-7.96 & 1893 & 12 & 134 & 14 & 0.00 & 0.00 & 0.00 & 0.00 & 5.60 & 0.63 \\
-4.75 & 1862 & 12 & 174 & 14 & 0.00 & 0.00 & 0.00 & 0.00 & 7.74 & 0.59 \\
-3.86 & 1872 & 6 & 114 & 4 & -0.05 & 0.04 & 0.26 & 0.05 & 7.30 & 0.37 \\
-1.73 & 1847 & 4 & 112 & 5 & 0.01 & 0.03 & 0.06 & 0.04 & 6.82 & 0.35 \\
-0.87 & 1849 & 5 & 129 & 6 & 0.05 & 0.03 & 0.01 & 0.04 & 6.70 & 0.29 \\
-0.36 & 1862 & 3 & 125 & 4 & 0.05 & 0.02 & 0.01 & 0.03 & 6.49 & 0.35 \\
-0.00 & 1862 & 4 & 132 & 4 & -0.02 & 0.02 & 0.02 & 0.03 & 6.91 & 0.34 \\
0.51 & 1864 & 4 & 127 & 5 & -0.03 & 0.03 & 0.04 & 0.03 & 6.86 & 0.29 \\
1.37 & 1865 & 4 & 108 & 4 & -0.03 & 0.03 & 0.13 & 0.03 & 7.88 & 0.35 \\
3.43 & 1864 & 6 & 134 & 6 & 0.01 & 0.04 & 0.08 & 0.04 & 6.61 & 0.38 \\
4.36 & 1862 & 10 & 146 & 11 & 0.00 & 0.00 & 0.00 & 0.00 & 6.17 & 0.62 \\
\hline \hline
\end{tabular}

Table A.3. NGC 5422

\begin{tabular}{ccccccccccc}
\hline \hline $\mathrm{x}\left[{ }^{\prime \prime}\right]$ & $\mathrm{V}$ & $\Delta V$ & $\sigma$ & $\Delta \sigma$ & $\mathrm{h}_{3}$ & $\Delta \mathrm{h}_{3}$ & $\mathrm{~h}_{4}$ & $\Delta \mathrm{h}_{4}$ & $\mathrm{CaT}^{*}$ & $\Delta \mathrm{CaT}^{*}$ \\
\hline-6.48 & 1841 & 13 & 144 & 15 & 0.00 & 0.00 & 0.00 & 0.00 & 5.56 & 0.62 \\
-4.86 & 1837 & 7 & 134 & 7 & -0.01 & 0.05 & 0.11 & 0.05 & 6.35 & 0.38 \\
-2.24 & 1854 & 5 & 138 & 5 & -0.02 & 0.03 & 0.06 & 0.03 & 6.82 & 0.36 \\
-1.23 & 1856 & 6 & 155 & 7 & 0.10 & 0.03 & -0.02 & 0.04 & 6.65 & 0.33 \\
-0.72 & 1839 & 7 & 176 & 7 & 0.09 & 0.03 & 0.02 & 0.04 & 7.01 & 0.36 \\
-0.36 & 1838 & 5 & 182 & 6 & 0.04 & 0.02 & -0.01 & 0.02 & 7.20 & 0.31 \\
-0.00 & 1832 & 5 & 187 & 7 & 0.00 & 0.02 & -0.06 & 0.03 & 6.68 & 0.30 \\
0.36 & 1844 & 7 & 190 & 8 & -0.05 & 0.03 & -0.00 & 0.03 & 6.25 & 0.35 \\
0.87 & 1827 & 7 & 183 & 7 & 0.03 & 0.03 & 0.08 & 0.03 & 7.18 & 0.32 \\
1.87 & 1830 & 7 & 165 & 7 & -0.02 & 0.03 & 0.06 & 0.04 & 6.70 & 0.36 \\
4.51 & 1841 & 7 & 131 & 9 & 0.01 & 0.05 & -0.01 & 0.06 & 6.14 & 0.38 \\
6.13 & 1843 & 11 & 103 & 13 & 0.00 & 0.00 & 0.00 & 0.00 & 4.61 & 0.65 \\
\hline \hline
\end{tabular}

Table A.4. NGC 5443

\begin{tabular}{ccccccccccc}
\hline \hline $\mathrm{x}\left[^{\prime \prime}\right]$ & $\mathrm{V}$ & $\Delta V$ & $\sigma$ & $\Delta \sigma$ & $\mathrm{h}_{3}$ & $\Delta \mathrm{h}_{3}$ & $\mathrm{~h}_{4}$ & $\Delta \mathrm{h}_{4}$ & $\mathrm{CaT}^{*}$ & $\Delta \mathrm{CaT}^{*}$ \\
\hline-7.47 & 1787 & 13 & 107 & 16 & 0.00 & 0.00 & 0.00 & 0.00 & 7.88 & 0.63 \\
-3.34 & 1739 & 9 & 110 & 11 & 0.00 & 0.00 & 0.00 & 0.00 & 4.69 & 0.61 \\
-1.74 & 1740 & 7 & 121 & 8 & 0.00 & 0.00 & 0.00 & 0.00 & 7.70 & 0.60 \\
-1.23 & 1738 & 5 & 115 & 6 & -0.01 & 0.04 & 0.03 & 0.05 & 7.66 & 0.37 \\
-0.02 & 1758 & 3 & 89 & 4 & -0.07 & 0.03 & 0.05 & 0.04 & 7.43 & 0.33 \\
1.67 & 1760 & 6 & 112 & 10 & 0.01 & 0.05 & -0.11 & 0.07 & 6.70 & 0.38 \\
4.36 & 1752 & 11 & 125 & 13 & 0.00 & 0.00 & 0.00 & 0.00 & 5.57 & 0.65 \\
\hline \hline
\end{tabular}


Table A.5. NGC 5475

\begin{tabular}{ccccccccccc}
\hline \hline $\mathrm{x}\left[{ }^{\prime \prime}\right]$ & $\mathrm{V}$ & $\Delta V$ & $\sigma$ & $\Delta \sigma$ & $\mathrm{h}_{3}$ & $\Delta \mathrm{h}_{3}$ & $\mathrm{~h}_{4}$ & $\Delta \mathrm{h}_{4}$ & $\mathrm{CaT}^{*}$ & $\Delta \mathrm{CaT}^{*}$ \\
\hline-4.12 & 1665 & 5 & 55 & 8 & 0.00 & 0.00 & 0.00 & 0.00 & 7.57 & 0.60 \\
-2.48 & 1665 & 5 & 74 & 6 & 0.00 & 0.00 & 0.00 & 0.00 & 6.94 & 0.60 \\
-1.50 & 1660 & 5 & 93 & 7 & 0.10 & 0.05 & 0.01 & 0.05 & 7.04 & 0.38 \\
-0.50 & 1669 & 5 & 111 & 7 & 0.03 & 0.04 & -0.09 & 0.06 & 6.91 & 0.30 \\
0.00 & 1661 & 3 & 97 & 4 & 0.02 & 0.03 & -0.02 & 0.04 & 7.52 & 0.36 \\
0.50 & 1674 & 6 & 91 & 7 & -0.05 & 0.05 & 0.04 & 0.06 & 7.40 & 0.32 \\
1.64 & 1667 & 5 & 89 & 6 & -0.01 & 0.05 & 0.06 & 0.05 & 7.32 & 0.37 \\
2.12 & 1665 & 5 & 87 & 7 & 0.00 & 0.00 & 0.00 & 0.00 & 6.65 & 0.55 \\
3.49 & 1671 & 4 & 43 & 6 & 0.00 & 0.00 & 0.00 & 0.00 & 7.33 & 0.61 \\
\hline \hline
\end{tabular}

Table A.6. NGC 5587

\begin{tabular}{ccccccccccc}
\hline \hline $\mathrm{x}\left[{ }^{\prime \prime}\right]$ & $\mathrm{V}$ & $\Delta V$ & $\sigma$ & $\Delta \sigma$ & $\mathrm{h}_{3}$ & $\Delta \mathrm{h}_{3}$ & $\mathrm{~h}_{4}$ & $\Delta \mathrm{h}_{4}$ & $\mathrm{CaT}^{*}$ & $\Delta \mathrm{CaT}^{*}$ \\
\hline-3.82 & 2291 & 6 & 76 & 9 & 0.00 & 0.00 & 0.00 & 0.00 & 6.66 & 0.62 \\
-1.43 & 2311 & 6 & 110 & 9 & -0.06 & 0.05 & -0.06 & 0.06 & 6.24 & 0.37 \\
-0.00 & 2296 & 3 & 100 & 4 & -0.03 & 0.03 & 0.06 & 0.03 & 7.09 & 0.35 \\
1.57 & 2280 & 6 & 79 & 6 & 0.19 & 0.07 & 0.09 & 0.06 & 7.95 & 0.37 \\
\hline \hline
\end{tabular}

Table A.7. NGC 5689

\begin{tabular}{ccccccccccc}
\hline \hline $\left.\mathrm{x}^{\prime \prime}\right]$ & $\mathrm{V}$ & $\Delta V$ & $\sigma$ & $\Delta \sigma$ & $\mathrm{h}_{3}$ & $\Delta \mathrm{h}_{3}$ & $\mathrm{~h}_{4}$ & $\Delta \mathrm{h}_{4}$ & $\mathrm{CaT}^{*}$ & $\Delta \mathrm{CaT}^{*}$ \\
\hline-9.04 & 2202 & 11 & 96 & 14 & 0.00 & 0.00 & 0.00 & 0.00 & 7.81 & 0.61 \\
-5.86 & 2173 & 9 & 137 & 10 & 0.00 & 0.00 & 0.00 & 0.00 & 6.57 & 0.62 \\
-4.10 & 2195 & 8 & 143 & 10 & 0.00 & 0.00 & 0.00 & 0.00 & 7.51 & 0.59 \\
-3.04 & 2202 & 5 & 138 & 5 & -0.04 & 0.03 & 0.11 & 0.04 & 6.87 & 0.37 \\
-1.37 & 2212 & 5 & 151 & 4 & -0.10 & 0.03 & 0.10 & 0.03 & 7.80 & 0.33 \\
-0.52 & 2214 & 4 & 157 & 5 & -0.07 & 0.02 & -0.05 & 0.03 & 6.84 & 0.28 \\
0.00 & 2200 & 5 & 158 & 5 & -0.04 & 0.02 & 0.02 & 0.03 & 6.44 & 0.36 \\
0.36 & 2201 & 5 & 163 & 5 & 0.01 & 0.02 & 0.01 & 0.03 & 7.48 & 0.37 \\
0.88 & 2192 & 5 & 154 & 5 & -0.03 & 0.02 & 0.04 & 0.03 & 6.88 & 0.30 \\
1.74 & 2184 & 5 & 158 & 5 & 0.04 & 0.02 & 0.07 & 0.03 & 7.78 & 0.33 \\
3.38 & 2200 & 6 & 147 & 7 & -0.05 & 0.04 & 0.05 & 0.04 & 7.05 & 0.38 \\
4.46 & 2211 & 8 & 100 & 10 & 0.00 & 0.00 & 0.00 & 0.00 & 5.70 & 0.62 \\
6.21 & 2209 & 9 & 125 & 11 & 0.00 & 0.00 & 0.00 & 0.00 & 5.76 & 0.63 \\
9.29 & 2218 & 9 & 92 & 11 & 0.00 & 0.00 & 0.00 & 0.00 & 7.72 & 0.62 \\
\hline \hline
\end{tabular}

Table A.8. NGC 5707

\begin{tabular}{ccccccccccc}
\hline \hline $\mathrm{x}\left[^{\prime \prime}\right]$ & $\mathrm{V}$ & $\Delta V$ & $\sigma$ & $\Delta \sigma$ & $\mathrm{h}_{3}$ & $\Delta \mathrm{h}_{3}$ & $\mathrm{~h}_{4}$ & $\Delta \mathrm{h}_{4}$ & $\mathrm{CaT}^{*}$ & $\Delta \mathrm{CaT}^{*}$ \\
\hline-5.06 & 2208 & 5 & 71 & 7 & 0.00 & 0.00 & 0.00 & 0.00 & 6.90 & 0.62 \\
-4.33 & 2211 & 5 & 85 & 5 & 0.02 & 0.05 & 0.14 & 0.05 & 7.36 & 0.37 \\
-1.89 & 2193 & 5 & 143 & 6 & 0.03 & 0.03 & -0.05 & 0.04 & 6.51 & 0.35 \\
-0.88 & 2179 & 6 & 154 & 8 & 0.01 & 0.03 & -0.00 & 0.04 & 7.33 & 0.34 \\
-0.01 & 2191 & 3 & 163 & 4 & -0.04 & 0.02 & -0.06 & 0.02 & 7.03 & 0.24 \\
1.01 & 2200 & 5 & 147 & 6 & -0.04 & 0.03 & 0.02 & 0.03 & 7.56 & 0.34 \\
2.66 & 2198 & 5 & 119 & 5 & -0.04 & 0.03 & 0.06 & 0.04 & 6.59 & 0.37 \\
3.20 & 2199 & 6 & 96 & 7 & 0.00 & 0.00 & 0.00 & 0.00 & 6.18 & 0.62 \\
4.83 & 2210 & 6 & 84 & 8 & 0.00 & 0.00 & 0.00 & 0.00 & 6.24 & 0.62 \\
\hline \hline
\end{tabular}


Table A.9. NGC 5719

\begin{tabular}{ccccccccccc}
\hline \hline $\mathrm{x}\left[{ }^{\prime \prime}\right]$ & $\mathrm{V}$ & $\Delta V$ & $\sigma$ & $\Delta \sigma$ & $\mathrm{h}_{3}$ & $\Delta \mathrm{h}_{3}$ & $\mathrm{~h}_{4}$ & $\Delta \mathrm{h}_{4}$ & CaT $^{*}$ & $\Delta \mathrm{CaT}^{*}$ \\
\hline-6.63 & 1731 & 8 & 82 & 10 & 0.00 & 0.00 & 0.00 & 0.00 & 7.93 & 0.62 \\
-4.44 & 1723 & 7 & 104 & 8 & 0.00 & 0.00 & 0.00 & 0.00 & 6.94 & 0.62 \\
-3.38 & 1719 & 4 & 83 & 5 & -0.01 & 0.04 & 0.25 & 0.04 & 7.39 & 0.37 \\
-1.75 & 1726 & 5 & 97 & 5 & -0.03 & 0.04 & 0.09 & 0.04 & 6.80 & 0.35 \\
-0.88 & 1719 & 4 & 102 & 4 & -0.02 & 0.03 & 0.17 & 0.03 & 6.86 & 0.33 \\
-0.01 & 1720 & 3 & 113 & 2 & 0.02 & 0.02 & 0.12 & 0.02 & 6.83 & 0.24 \\
0.87 & 1717 & 5 & 125 & 6 & 0.02 & 0.03 & 0.02 & 0.04 & 7.30 & 0.36 \\
2.12 & 1723 & 5 & 92 & 5 & -0.02 & 0.04 & 0.13 & 0.04 & 7.11 & 0.37 \\
3.89 & 1728 & 5 & 72 & 7 & 0.00 & 0.00 & 0.00 & 0.00 & 7.62 & 0.58 \\
8.01 & 1712 & 15 & 57 & 21 & 0.00 & 0.00 & 0.00 & 0.00 & 7.28 & 0.70 \\
\hline \hline
\end{tabular}

Table A.10. NGC 5746

\begin{tabular}{ccccccccccc}
\hline \hline $\mathrm{x}\left[^{\prime \prime}\right]$ & $\mathrm{V}$ & $\Delta V$ & $\sigma$ & $\Delta \sigma$ & $\mathrm{h}_{3}$ & $\Delta \mathrm{h}_{3}$ & $\mathrm{~h}_{4}$ & $\Delta \mathrm{h}_{4}$ & $\mathrm{CaT}^{*}$ & $\Delta \mathrm{CaT}^{*}$ \\
\hline-13.66 & 1690 & 15 & 161 & 17 & 0.00 & 0.00 & 0.00 & 0.00 & 5.49 & 0.62 \\
-11.52 & 1703 & 11 & 164 & 13 & 0.02 & 0.05 & -0.01 & 0.06 & 5.61 & 0.38 \\
-6.14 & 1719 & 9 & 223 & 10 & -0.01 & 0.03 & -0.02 & 0.04 & 6.43 & 0.37 \\
-3.32 & 1713 & 7 & 215 & 7 & -0.03 & 0.03 & 0.05 & 0.03 & 6.53 & 0.35 \\
-1.76 & 1711 & 7 & 184 & 8 & 0.01 & 0.03 & 0.04 & 0.03 & 6.70 & 0.37 \\
-0.89 & 1713 & 5 & 161 & 5 & -0.05 & 0.03 & 0.04 & 0.03 & 7.04 & 0.37 \\
-0.00 & 1723 & 4 & 160 & 5 & -0.04 & 0.02 & 0.01 & 0.03 & 6.86 & 0.28 \\
1.03 & 1714 & 6 & 182 & 5 & 0.00 & 0.03 & 0.10 & 0.03 & 7.19 & 0.32 \\
2.43 & 1691 & 7 & 228 & 7 & -0.02 & 0.02 & 0.06 & 0.03 & 6.74 & 0.36 \\
6.19 & 1733 & 12 & 202 & 14 & -0.06 & 0.05 & -0.03 & 0.05 & 5.15 & 0.39 \\
7.14 & 1714 & 18 & 175 & 20 & 0.00 & 0.00 & 0.00 & 0.00 & 4.62 & 0.70 \\
12.51 & 1727 & 18 & 184 & 21 & 0.00 & 0.00 & 0.00 & 0.00 & 5.47 & 0.68 \\
\hline \hline
\end{tabular}

Table A.11. NGC 5838

\begin{tabular}{ccccccccccc}
\hline \hline $\left.\mathrm{x}^{\left.{ }^{\prime}\right]}\right]$ & $\mathrm{V}$ & $\Delta V$ & $\sigma$ & $\Delta \sigma$ & $\mathrm{h}_{3}$ & $\Delta \mathrm{h}_{3}$ & $\mathrm{~h}_{4}$ & $\Delta \mathrm{h}_{4}$ & $\mathrm{CaT}^{*}$ & $\Delta \mathrm{CaT}^{*}$ \\
\hline-14.62 & 1358 & 16 & 172 & 11 & 0.13 & 0.09 & 0.32 & 0.11 & 8.43 & 0.38 \\
-7.00 & 1341 & 8 & 172 & 8 & 0.03 & 0.04 & 0.06 & 0.04 & 7.04 & 0.37 \\
-4.61 & 1345 & 9 & 220 & 9 & 0.05 & 0.03 & 0.06 & 0.04 & 6.35 & 0.35 \\
-3.21 & 1369 & 9 & 282 & 9 & -0.03 & 0.02 & 0.05 & 0.03 & 6.86 & 0.36 \\
-2.32 & 1332 & 9 & 267 & 12 & 0.03 & 0.03 & -0.06 & 0.03 & 6.73 & 0.36 \\
-1.60 & 1336 & 8 & 267 & 10 & 0.03 & 0.02 & -0.04 & 0.03 & 6.49 & 0.29 \\
-1.08 & 1325 & 11 & 302 & 12 & -0.01 & 0.03 & 0.01 & 0.03 & 6.35 & 0.33 \\
-0.72 & 1328 & 8 & 271 & 9 & 0.03 & 0.02 & -0.02 & 0.03 & 7.01 & 0.29 \\
-0.36 & 1336 & 7 & 278 & 9 & -0.01 & 0.02 & -0.00 & 0.02 & 6.80 & 0.26 \\
0.00 & 1345 & 7 & 291 & 8 & 0.03 & 0.02 & 0.00 & 0.02 & 6.51 & 0.25 \\
0.36 & 1382 & 8 & 319 & 10 & 0.04 & 0.02 & -0.03 & 0.02 & 7.81 & 0.25 \\
0.72 & 1381 & 10 & 295 & 11 & -0.11 & 0.03 & -0.03 & 0.03 & 6.37 & 0.27 \\
1.08 & 1398 & 11 & 271 & 13 & -0.05 & 0.03 & -0.06 & 0.04 & 6.59 & 0.32 \\
1.44 & 1365 & 11 & 322 & 14 & -0.05 & 0.03 & -0.03 & 0.03 & 7.01 & 0.37 \\
1.96 & 1359 & 10 & 295 & 9 & -0.00 & 0.03 & 0.09 & 0.03 & 6.86 & 0.30 \\
2.68 & 1360 & 10 & 244 & 10 & -0.07 & 0.03 & 0.08 & 0.04 & 6.90 & 0.35 \\
3.57 & 1298 & 10 & 278 & 10 & 0.03 & 0.03 & 0.06 & 0.03 & 6.53 & 0.35 \\
4.81 & 1331 & 10 & 227 & 12 & -0.05 & 0.04 & -0.04 & 0.04 & 5.79 & 0.38 \\
6.71 & 1322 & 9 & 212 & 8 & 0.03 & 0.03 & 0.09 & 0.04 & 7.15 & 0.38 \\
11.00 & 1327 & 16 & 160 & 22 & 0.02 & 0.08 & -0.10 & 0.11 & 5.26 & 0.39 \\
11.74 & 1353 & 21 & 157 & 24 & 0.00 & 0.00 & 0.00 & 0.00 & 3.65 & 0.62 \\
\hline \hline
\end{tabular}


Table A.12. NGC 5854

\begin{tabular}{ccccccccccc}
\hline \hline $\mathrm{x}\left[^{\prime \prime}\right]$ & $\mathrm{V}$ & $\Delta V$ & $\sigma$ & $\Delta \sigma$ & $\mathrm{h}_{3}$ & $\Delta \mathrm{h}_{3}$ & $\mathrm{~h}_{4}$ & $\Delta \mathrm{h}_{4}$ & $\mathrm{CaT}^{*}$ & $\Delta \mathrm{CaT}^{*}$ \\
\hline-5.98 & 1682 & 8 & 103 & 9 & 0.00 & 0.00 & 0.00 & 0.00 & 7.97 & 0.60 \\
-4.31 & 1677 & 5 & 67 & 4 & 0.01 & 0.06 & 0.34 & 0.06 & 7.46 & 0.37 \\
-1.88 & 1660 & 4 & 95 & 6 & 0.05 & 0.04 & -0.05 & 0.05 & 6.47 & 0.37 \\
-0.86 & 1659 & 5 & 100 & 6 & 0.05 & 0.04 & -0.03 & 0.05 & 5.96 & 0.31 \\
-0.36 & 1675 & 4 & 107 & 5 & -0.00 & 0.03 & -0.00 & 0.04 & 6.78 & 0.35 \\
0.00 & 1670 & 3 & 102 & 4 & 0.02 & 0.03 & 0.05 & 0.03 & 6.73 & 0.33 \\
0.36 & 1676 & 3 & 121 & 5 & -0.03 & 0.02 & -0.04 & 0.03 & 7.75 & 0.33 \\
0.86 & 1698 & 4 & 108 & 4 & -0.10 & 0.03 & 0.07 & 0.04 & 7.88 & 0.30 \\
1.88 & 1677 & 4 & 121 & 6 & 0.05 & 0.03 & -0.03 & 0.04 & 7.65 & 0.36 \\
4.31 & 1659 & 5 & 74 & 5 & 0.01 & 0.05 & 0.10 & 0.05 & 6.55 & 0.38 \\
5.98 & 1660 & 6 & 73 & 8 & 0.00 & 0.00 & 0.00 & 0.00 & 5.55 & 0.61 \\
\hline \hline
\end{tabular}

Table A.13. NGC 5879

\begin{tabular}{ccccccccccc}
\hline \hline $\left.\mathrm{x}^{\prime \prime}\right]$ & $\mathrm{V}$ & $\Delta V$ & $\sigma$ & $\Delta \sigma$ & $\mathrm{h}_{3}$ & $\Delta \mathrm{h}_{3}$ & $\mathrm{~h}_{4}$ & $\Delta \mathrm{h}_{4}$ & $\mathrm{CaT}^{*}$ & $\Delta \mathrm{CaT}^{*}$ \\
\hline-7.00 & 764 & 10 & 70 & 14 & 0.00 & 0.00 & 0.00 & 0.00 & 4.88 & 0.63 \\
-4.24 & 785 & 4 & 51 & 6 & 0.00 & 0.00 & 0.00 & 0.00 & 6.44 & 0.60 \\
-2.68 & 777 & 5 & 43 & 8 & 0.06 & 0.10 & 0.10 & 0.12 & 6.35 & 0.38 \\
-1.03 & 780 & 5 & 68 & 6 & 0.04 & 0.06 & 0.02 & 0.07 & 6.88 & 0.36 \\
-0.01 & 779 & 3 & 51 & 3 & 0.00 & 0.04 & 0.22 & 0.04 & 6.47 & 0.30 \\
1.17 & 781 & 4 & 45 & 5 & -0.00 & 0.07 & 0.22 & 0.07 & 7.55 & 0.34 \\
4.30 & 780 & 14 & 34 & 22 & 0.00 & 0.30 & 0.24 & 0.33 & 6.55 & 0.38 \\
6.42 & 776 & 10 & 41 & 15 & 0.00 & 0.00 & 0.00 & 0.00 & 4.06 & 0.63 \\
\hline \hline
\end{tabular}

Table A.14. NGC 5965

\begin{tabular}{ccccccccccc}
\hline \hline $\mathrm{x}\left[{ }^{\prime \prime}\right]$ & $\mathrm{V}$ & $\Delta V$ & $\sigma$ & $\Delta \sigma$ & $\mathrm{h}_{3}$ & $\Delta \mathrm{h}_{3}$ & $\mathrm{~h}_{4}$ & $\Delta \mathrm{h}_{4}$ & $\mathrm{CaT}^{*}$ & $\Delta \mathrm{CaT}^{*}$ \\
\hline-5.41 & 3375 & 12 & 148 & 14 & 0.00 & 0.00 & 0.00 & 0.00 & 4.80 & 0.62 \\
-3.08 & 3398 & 9 & 192 & 8 & 0.11 & 0.04 & 0.08 & 0.04 & 5.22 & 0.38 \\
-1.00 & 3411 & 9 & 160 & 7 & -0.01 & 0.05 & 0.19 & 0.06 & 6.18 & 0.37 \\
0.00 & 3417 & 5 & 191 & 6 & -0.01 & 0.02 & 0.05 & 0.03 & 7.37 & 0.27 \\
0.99 & 3421 & 10 & 189 & 14 & 0.05 & 0.05 & -0.09 & 0.06 & 7.27 & 0.38 \\
2.22 & 3368 & 12 & 170 & 15 & 0.00 & 0.00 & 0.00 & 0.00 & 5.19 & 0.61 \\
\hline \hline
\end{tabular}

Table A.15. NGC 6010

\begin{tabular}{ccccccccccc}
\hline \hline $\left.\mathrm{x}^{\prime \prime}\right]$ & $\mathrm{V}$ & $\Delta V$ & $\sigma$ & $\Delta \sigma$ & $\mathrm{h}_{3}$ & $\Delta \mathrm{h}_{3}$ & $\mathrm{~h}_{4}$ & $\Delta \mathrm{h}_{4}$ & $\mathrm{CaT}^{*}$ & $\Delta \mathrm{CaT}^{*}$ \\
\hline-6.51 & 2038 & 22 & 110 & 26 & 0.00 & 0.00 & 0.00 & 0.00 & 7.96 & 0.67 \\
-3.70 & 2028 & 9 & 105 & 11 & 0.00 & 0.00 & 0.00 & 0.00 & 7.76 & 0.60 \\
-2.68 & 2038 & 8 & 123 & 10 & 0.00 & 0.00 & 0.00 & 0.00 & 8.27 & 0.61 \\
-2.23 & 2027 & 7 & 130 & 7 & -0.06 & 0.04 & 0.09 & 0.05 & 7.67 & 0.38 \\
-1.23 & 2013 & 8 & 150 & 9 & -0.06 & 0.04 & -0.03 & 0.05 & 7.61 & 0.34 \\
-0.72 & 1991 & 9 & 160 & 8 & -0.15 & 0.05 & 0.04 & 0.05 & 7.02 & 0.37 \\
-0.36 & 2010 & 5 & 156 & 5 & -0.10 & 0.03 & 0.03 & 0.03 & 7.05 & 0.32 \\
-0.00 & 2020 & 4 & 163 & 5 & -0.02 & 0.02 & -0.02 & 0.02 & 6.21 & 0.31 \\
0.36 & 2025 & 7 & 177 & 9 & -0.06 & 0.03 & -0.08 & 0.04 & 6.33 & 0.35 \\
0.87 & 2036 & 7 & 170 & 8 & -0.02 & 0.03 & 0.02 & 0.04 & 7.23 & 0.32 \\
1.87 & 2015 & 5 & 139 & 6 & 0.05 & 0.03 & 0.04 & 0.04 & 7.12 & 0.36 \\
2.83 & 2017 & 8 & 119 & 9 & 0.00 & 0.00 & 0.00 & 0.00 & 6.64 & 0.58 \\
4.47 & 2030 & 11 & 84 & 14 & 0.00 & 0.00 & 0.00 & 0.00 & 7.36 & 0.61 \\
\hline \hline
\end{tabular}


J. Falcón-Barroso et al.: Minor axis kinematics of 19 S0-Sbc bulges, Online Material p 7

Table A.16. NGC 6504

\begin{tabular}{ccccccccccc}
\hline \hline $\mathrm{x}^{\left.{ }^{\prime \prime}\right]}$ & $\mathrm{V}$ & $\Delta V$ & $\sigma$ & $\Delta \sigma$ & $\mathrm{h}_{3}$ & $\Delta \mathrm{h}_{3}$ & $\mathrm{~h}_{4}$ & $\Delta \mathrm{h}_{4}$ & CaT $^{*}$ & $\Delta \mathrm{CaT}^{*}$ \\
\hline-4.01 & 4724 & 13 & 191 & 16 & 0.00 & 0.00 & 0.00 & 0.00 & 6.38 & 0.61 \\
-3.12 & 4725 & 8 & 195 & 8 & 0.03 & 0.03 & 0.12 & 0.04 & 7.00 & 0.38 \\
-0.99 & 4740 & 11 & 248 & 14 & -0.00 & 0.04 & -0.05 & 0.04 & 7.47 & 0.34 \\
0.01 & 4735 & 5 & 213 & 5 & 0.00 & 0.02 & 0.07 & 0.02 & 7.15 & 0.24 \\
0.87 & 4760 & 8 & 180 & 8 & -0.09 & 0.04 & 0.07 & 0.04 & 6.07 & 0.35 \\
2.00 & 4732 & 8 & 191 & 9 & 0.01 & 0.03 & 0.03 & 0.04 & 5.99 & 0.37 \\
2.48 & 4739 & 10 & 187 & 12 & 0.00 & 0.00 & 0.00 & 0.00 & 6.18 & 0.55 \\
3.86 & 4722 & 12 & 203 & 15 & 0.00 & 0.00 & 0.00 & 0.00 & 7.71 & 0.59 \\
\hline \hline
\end{tabular}

Table A.17. NGC 7331

\begin{tabular}{ccccccccccc}
\hline \hline $\mathrm{x}^{\left.{ }^{\prime}\right]}$ & $\mathrm{V}$ & $\Delta V$ & $\sigma$ & $\Delta \sigma$ & $\mathrm{h}_{3}$ & $\Delta \mathrm{h}_{3}$ & $\mathrm{~h}_{4}$ & $\Delta \mathrm{h}_{4}$ & $\mathrm{CaT}^{*}$ & $\Delta \mathrm{CaT}^{*}$ \\
\hline-22.43 & 771 & 25 & 142 & 29 & 0.00 & 0.00 & 0.00 & 0.00 & 7.15 & 0.83 \\
-19.02 & 781 & 11 & 93 & 14 & 0.00 & 0.00 & 0.00 & 0.00 & 6.86 & 0.64 \\
-17.32 & 807 & 9 & 85 & 9 & -0.11 & 0.09 & 0.34 & 0.09 & 5.93 & 0.37 \\
-13.03 & 804 & 8 & 115 & 7 & -0.04 & 0.06 & 0.17 & 0.06 & 6.38 & 0.37 \\
-10.21 & 816 & 6 & 97 & 5 & -0.01 & 0.05 & 0.27 & 0.05 & 7.34 & 0.37 \\
-8.25 & 810 & 5 & 88 & 4 & 0.01 & 0.05 & 0.27 & 0.05 & 6.19 & 0.36 \\
-6.64 & 811 & 5 & 103 & 4 & -0.11 & 0.04 & 0.19 & 0.04 & 6.66 & 0.36 \\
-5.38 & 816 & 5 & 118 & 5 & -0.09 & 0.04 & 0.15 & 0.04 & 7.53 & 0.37 \\
-4.30 & 822 & 4 & 117 & 4 & -0.02 & 0.03 & 0.16 & 0.03 & 7.26 & 0.32 \\
-3.41 & 834 & 4 & 112 & 4 & -0.12 & 0.03 & 0.09 & 0.03 & 6.91 & 0.34 \\
-2.69 & 828 & 3 & 118 & 3 & -0.04 & 0.02 & 0.15 & 0.03 & 6.27 & 0.30 \\
-2.16 & 846 & 4 & 108 & 3 & -0.05 & 0.03 & 0.20 & 0.03 & 7.39 & 0.37 \\
-1.80 & 827 & 4 & 116 & 6 & -0.01 & 0.03 & -0.07 & 0.05 & 6.60 & 0.34 \\
-1.44 & 835 & 4 & 129 & 5 & 0.08 & 0.03 & -0.05 & 0.04 & 6.65 & 0.30 \\
-1.08 & 821 & 3 & 131 & 5 & -0.03 & 0.02 & -0.04 & 0.03 & 6.50 & 0.26 \\
-0.72 & 817 & 3 & 137 & 4 & 0.02 & 0.02 & -0.04 & 0.03 & 6.54 & 0.22 \\
-0.36 & 821 & 3 & 135 & 3 & -0.03 & 0.02 & 0.01 & 0.02 & 7.06 & 0.18 \\
-0.00 & 821 & 2 & 138 & 3 & -0.02 & 0.01 & 0.03 & 0.02 & 7.65 & 0.18 \\
0.36 & 833 & 3 & 136 & 3 & -0.04 & 0.02 & 0.08 & 0.02 & 7.37 & 0.20 \\
0.72 & 830 & 3 & 139 & 4 & -0.05 & 0.02 & 0.02 & 0.02 & 7.60 & 0.24 \\
1.08 & 828 & 5 & 146 & 5 & 0.01 & 0.03 & 0.10 & 0.03 & 8.43 & 0.29 \\
1.44 & 817 & 4 & 120 & 4 & 0.06 & 0.03 & 0.14 & 0.03 & 7.07 & 0.35 \\
1.96 & 820 & 4 & 120 & 4 & 0.01 & 0.03 & 0.09 & 0.03 & 6.78 & 0.30 \\
2.70 & 814 & 4 & 118 & 4 & -0.04 & 0.03 & 0.09 & 0.03 & 7.18 & 0.34 \\
3.41 & 808 & 4 & 95 & 5 & 0.09 & 0.04 & 0.06 & 0.04 & 6.52 & 0.37 \\
4.29 & 826 & 5 & 120 & 5 & -0.06 & 0.03 & 0.11 & 0.04 & 6.76 & 0.36 \\
5.67 & 802 & 5 & 115 & 5 & -0.02 & 0.04 & 0.13 & 0.04 & 7.29 & 0.36 \\
7.89 & 806 & 7 & 112 & 6 & -0.15 & 0.05 & 0.15 & 0.05 & 6.23 & 0.36 \\
11.47 & 794 & 12 & 103 & 9 & -0.30 & 0.12 & 0.25 & 0.11 & 6.27 & 0.40 \\
15.74 & 704 & 39 & 133 & 46 & 0.00 & 0.00 & 0.00 & 0.00 & 12.04 & 1.21 \\
\hline \hline & & & & & & & & & &
\end{tabular}


J. Falcón-Barroso et al.: Minor axis kinematics of 19 S0-Sbc bulges, Online Material $p 8$

Table A.18. NGC 7332

\begin{tabular}{ccccccccccc}
\hline \hline $\mathrm{x}\left[^{\prime \prime}\right]$ & $\mathrm{V}$ & $\Delta V$ & $\sigma$ & $\Delta \sigma$ & $\mathrm{h}_{3}$ & $\Delta \mathrm{h}_{3}$ & $\mathrm{~h}_{4}$ & $\Delta \mathrm{h}_{4}$ & $\mathrm{CaT}^{*}$ & $\Delta \mathrm{CaT}^{*}$ \\
\hline-12.21 & 1214 & 13 & 57 & 19 & 0.00 & 0.00 & 0.00 & 0.00 & 3.47 & 0.64 \\
-8.17 & 1224 & 8 & 93 & 10 & 0.00 & 0.00 & 0.00 & 0.00 & 6.41 & 0.60 \\
-6.78 & 1228 & 5 & 88 & 6 & -0.08 & 0.05 & 0.06 & 0.05 & 6.53 & 0.37 \\
-3.87 & 1214 & 4 & 110 & 5 & 0.04 & 0.03 & -0.03 & 0.04 & 5.55 & 0.38 \\
-2.47 & 1219 & 4 & 120 & 5 & -0.04 & 0.03 & -0.01 & 0.04 & 6.10 & 0.36 \\
-1.59 & 1231 & 6 & 122 & 7 & 0.03 & 0.05 & 0.03 & 0.05 & 8.05 & 0.31 \\
-1.08 & 1237 & 4 & 126 & 5 & -0.02 & 0.03 & 0.01 & 0.03 & 7.12 & 0.33 \\
-0.72 & 1230 & 3 & 125 & 4 & -0.01 & 0.02 & 0.06 & 0.03 & 6.77 & 0.26 \\
-0.36 & 1230 & 3 & 131 & 3 & 0.01 & 0.02 & 0.05 & 0.02 & 7.56 & 0.22 \\
0.00 & 1227 & 2 & 130 & 3 & 0.05 & 0.02 & -0.03 & 0.02 & 6.85 & 0.21 \\
0.36 & 1223 & 2 & 127 & 3 & 0.02 & 0.02 & 0.04 & 0.02 & 7.62 & 0.21 \\
0.72 & 1227 & 3 & 123 & 3 & -0.05 & 0.02 & 0.02 & 0.02 & 7.04 & 0.22 \\
1.08 & 1225 & 3 & 122 & 4 & -0.02 & 0.02 & 0.01 & 0.03 & 7.14 & 0.25 \\
1.44 & 1227 & 4 & 133 & 5 & -0.01 & 0.03 & -0.03 & 0.03 & 7.01 & 0.32 \\
1.95 & 1222 & 3 & 116 & 4 & -0.01 & 0.03 & -0.01 & 0.03 & 6.57 & 0.31 \\
2.83 & 1223 & 5 & 121 & 5 & 0.02 & 0.03 & 0.03 & 0.04 & 6.56 & 0.36 \\
4.23 & 1230 & 4 & 99 & 4 & 0.03 & 0.04 & 0.07 & 0.04 & 6.61 & 0.38 \\
7.14 & 1214 & 5 & 94 & 6 & 0.03 & 0.05 & 0.09 & 0.05 & 6.56 & 0.37 \\
8.53 & 1208 & 7 & 78 & 10 & 0.00 & 0.00 & 0.00 & 0.00 & 5.13 & 0.61 \\
12.46 & 1223 & 11 & 99 & 14 & 0.00 & 0.00 & 0.00 & 0.00 & 5.26 & 0.62 \\
\hline \hline
\end{tabular}

Table A.19. NGC 7457

\begin{tabular}{ccccccccccc}
\hline \hline $\mathrm{x}\left[^{\prime \prime}\right]$ & $\mathrm{V}$ & $\Delta V$ & $\sigma$ & $\Delta \sigma$ & $\mathrm{h}_{3}$ & $\Delta \mathrm{h}_{3}$ & $\mathrm{~h}_{4}$ & $\Delta \mathrm{h}_{4}$ & $\mathrm{CaT}^{*}$ & $\Delta \mathrm{CaT}^{*}$ \\
\hline-11.00 & 833 & 6 & 45 & 9 & 0.00 & 0.00 & 0.00 & 0.00 & 4.68 & 0.63 \\
-5.98 & 837 & 4 & 60 & 6 & 0.00 & 0.00 & 0.00 & 0.00 & 6.69 & 0.62 \\
-3.31 & 842 & 3 & 59 & 4 & 0.02 & 0.04 & 0.03 & 0.05 & 6.96 & 0.37 \\
-1.00 & 847 & 4 & 59 & 6 & -0.02 & 0.05 & -0.02 & 0.07 & 7.56 & 0.37 \\
0.01 & 839 & 2 & 59 & 2 & -0.02 & 0.03 & 0.05 & 0.03 & 7.70 & 0.24 \\
0.88 & 836 & 3 & 66 & 3 & 0.03 & 0.03 & 0.05 & 0.04 & 7.88 & 0.32 \\
1.85 & 840 & 3 & 71 & 4 & 0.08 & 0.04 & -0.01 & 0.04 & 7.20 & 0.37 \\
4.97 & 848 & 4 & 52 & 5 & -0.07 & 0.06 & 0.02 & 0.07 & 7.15 & 0.38 \\
6.68 & 853 & 4 & 47 & 6 & 0.00 & 0.00 & 0.00 & 0.00 & 7.24 & 0.61 \\
11.63 & 851 & 6 & 54 & 8 & 0.00 & 0.00 & 0.00 & 0.00 & 7.15 & 0.62 \\
\hline \hline
\end{tabular}

